\title{
On the coupling between magnetic field and nutation in a numerical integration approach
}

\author{
C.-L. Huang, ${ }^{1}$ V. Dehant, ${ }^{2}$ X.-H. Liao, ${ }^{1}$ T. Van Hoolst, ${ }^{2}$ and M. G. Rochester ${ }^{3}$ \\ Received 19 May 2010; revised 6 January 2011; accepted 12 January 2011; published 30 March 2011.
}

[1] Nutation amplitudes are computed in a displacement field approach that incorporates the influence of a prescribed magnetic field inside the Earth's core. The existence of relative nutational motions between the liquid core and its surrounding solid parts induces a shearing of the magnetic field. An incremental magnetic field is then created, which in return perturbs the nutations themselves. This problem has already been addressed within a nutation model computed from an angular momentum budget approach. Here we incorporate the magnetic field influence directly in the motion equation and in the boundary conditions used in precise nutation theory, and a new strategy to compute nutations is established. As in previous studies, we assume that the root-mean-square of the radial magnetic field amplitude at the core-mantle boundary is 6.9 Gauss, that the magnetic diffusivity at the bottom of the mantle and in the fluid outer core side is $1.6 \mathrm{~m}^{2} / \mathrm{s}$, and that the thickness of the conductive layer at the bottom of the mantle is $200 \mathrm{~m}$.

The Coriolis force is included in this work. The results show that the free core nutation period decreases by 0.38 days, and that the out-of-phase (in-phase) amplitudes of the retrograde 18.6 year and the retrograde annual nutations increase (decrease) by 20 and $39 \mu a s$, respectively. Comparisons of these results with previous studies are made, and discussions are also presented on the contribution of Coriolis force and the prescribed magnetic field on the coupling constants.

Citation: Huang, C.-L., V. Dehant, X.-H. Liao, T. Van Hoolst, and M. G. Rochester (2011), On the coupling between magnetic field and nutation in a numerical integration approach, J. Geophys. Res., 116, B03403, doi:10.1029/2010JB007713.

\section{Introduction}

[2] Precession and nutation are secular and long periodic motions (periods longer than 2 days) of the Celestial Intermediate Pole (CIP) in space. The CIP is the direction of a conceptual axis describing the same motion as the Earth's rotation axis in space for periods longer than 2 days [Capitaine et al., 2003], and the same motion as the Earth's rotation axis inside the Earth except for the retrograde motion of quasi-diurnal periods. The nutation model adopted by the International Astronomical Union (IAU) in 2000 and the International Union of Geodesy and Geophysics (IUGG) in 2003 is the so-called MHB2000 model.

[3] Based on the angular momentum approach of Sasao et al. [1980], Mathews et al. [1991] presented a semianalytical calculation of the Earth's transfer function for nutations. In the work of Mathews et al. [1991], a deformable solid inner core (SIC), a fluid outer core (FOC), and an elastic mantle are considered, and 9 compliance parameters in the

\footnotetext{
${ }^{1}$ Shanghai Astronomical Observatory, Chinese Academy of Sciences, Shanghai, China.

${ }^{2}$ Royal Observatory of Belgium, Brussels, Belgium.

${ }^{3}$ Department of Earth Sciences, Memorial University of Newfoundland, St. John's, Newfoundland and Labrador, Canada.

Copyright 2011 by the American Geophysical Union. 0148-0227/11/2010JB007713
}

formulae are to be fitted from VLBI observations [Herring et al., 1991]. In order to minimize the residuals on the retrograde annual ( -1 year) nutation term, Buffett [1992, hereafter paper 1] and Buffett et al. [1993] introduced a fitted constant in the only mechanism of coupling they considered, between the magnetic field and nutation at the core-mantle boundary $(\mathrm{CMB})$. After one decade, these authors updated their model in three new papers [Mathews et al., 2002; Buffett et al., 2002, hereafter paper 2; Herring et al., 2002]. In this new model (MHB2000), mantle anelasticity, ocean and atmospheric effects are incorporated, more VLBI observation data of better precision are used and, moreover, the computation of the coupling between magnetic field and nutation at $\mathrm{CMB}$ (and also at the boundary between the inner core and the outer core, ICB) is improved. However, it is only recently that there are a few papers discussing the MHB theory itself [e.g., Rochester and Crossley, 2009].

[4] Several other available theoretical nutation models of the nonrigid Earth have been published recently. Since 1995, a Spanish group has been working on a new nutation theory and published a series of papers improving the nutation model by a generalization of the Hamiltonian method accounting for the nonrigid Earth [see, e.g., Getino, 1995; Getino and Ferrándiz, 2001]. Using a numerical integration approach first proposed by Smith [1974] (see also Wahr [1981] or Rogister [2001]), the Royal Observatory of Belgium group has studied new nutation models for more than one decade, 
incorporating mantle anelasticity, nonhydrostatic contributions from mantle lateral heterogeneities in density and nonhydrostatic boundaries [see, e.g., Dehant, 1990; Dehant and Defraigne, 1997]. Due to the resonance of nutation with the free core nutation (FCN), the period of FCN plays a key role in nutation amplitudes, and the FCN related parameters, such as a change in the core flattening and various dissipation factors (like magnetic, viscous, and topographic dissipation) in the Earth's interior, are important for the nutation models. Therefore, besides including the contributions from the ocean tides and the atmospheric angular momentum via the outer surface nonfree boundary conditions, Schastok [1997] and Huang et al. [2001] have paid special attention to the boundary conditions at the $\mathrm{CMB}$ by introducing a thin nonhydrostatic equilibrium layer there, expanding these boundary conditions to the second order of ellipticity [Huang, 2001], and modifying the ellipticity of the $\mathrm{CMB}$ a little $(4 \sim 5 \%)$ by fitting to the observed FCN period.

[5] On the one hand, all the above theoretical nutation models are very comparable to each other although they use different approaches; on the other hand, the most important unsolved problem in the above nutation models is the observed differences for the principal nutation terms (mainly the -1 year term, the 18.6 year terms, and the prograde semiannual term) between the theoretical amplitudes based on an Earth model and the VLBI observations.

[6] One significant improvement of MHB2000 over the other models is that the observed differences in the principal nutation terms (mainly the -1 year term) with respect to VLBI observations are removed mostly by accounting for the effects of electromagnetic coupling (EMC) of the liquid core to the mantle and the inner core. In MHB2000, the coupling constants at $\mathrm{CMB}$ and ICB and related compliance parameters appearing in the incremental products of inertia are fitted to the VLBI nutation observation data.

[7] In the original work of Rochester and Smylie [1965], the equatorial components of the electromagnetic torque act on the mantle is calculated rigorously and the electromagnetic damping of the Chandler wobble is first quantitatively investigated, and it is shown that the electromagnetic core-mantle coupling fails by several orders of magnitude either to generate or to damp the Chandler wobble. Following the same way of this work, paper 1 and paper 2 also consider that this coupling is a consequence of the Lorentz forces induced by the interaction between the global static magnetic field that crosses the boundaries and the relative rotation of the outer core with respect to the mantle or to the inner core. The mantle is generally considered as poorly conducting, but it is supposed that there exists a highly conducting layer at the bottom of the mantle, possibly due to chemical reactions between the mantle silicates and the conducting liquid iron alloy [Buffett et al., 2000; Buffett, 2007]. This layer hosts the induced magnetic field, which decreases very sharply through the thin layer and vanishes in the bulk of the mantle. The relative rotation between the mantle and the outer core (and equivalently between the outer core and the inner core) distorts the magnetic field near the core boundaries, which in return, induces changes in the differential rotations and thus in the nutations. From this description, it is easy to see that the nutation amplitudes and the magnetic field are coupled together and that a first-order computation is probably sufficient.
[8] Meanwhile, there are many papers which discuss the possible effects of EMC at the CMB on other features of the Earth's rotation than nutation, including the secular decrease of obliquity [e.g., Rochester, 1976] and the decade variation of length of day [e.g., Holme, 1998, 2000; Stix and Roberts, 1984; Wicht and Jault, 1999]. EMC is also assumed as a source of dissipation for torsional oscillation [e.g., Dumberry and Mound, 2008; Buffett et al., 2009]. The details of these discussion are outside the scope of this paper and readers are referred to the respective papers.

[9] The purpose of this paper is to introduce the necessary theoretical developments in the numerical integration method and to compute the nutation perturbations induced by considering a prescribed static electromagnetic field. This paper follows the approach and the notation defined by Smith [1974]. The displacement field approach in essence considers coupling between the toroidal nutational motion and the other components of the displacement field within a rotating ellipsoidal Earth. It offers the advantage of working in a completely consistent displacement field and thus opens interesting perspectives. We calculate the influence of the magnetic field on nutations and compare with the results from the MHB2000 approach. Except for the particularities of the approaches, if the same approximations are considered, one should retrieve similar results as paper 1 and paper 2 .

[10] This paper is organized as follows. In section 2, we present the dynamic equations for nutation and the induction equation for the magnetic field and explain how the introduction of the static magnetic field is treated in the frame of a numerical integration method for computing nutations (and tides). We also develop a strategy to deal with the Lorentz force in the numerical integration approach as well as new boundary conditions and apply them to the particular case of the approximations used in paper 1 . In section 3 , we present the results, discussion, short summary and remarks. Related notes are given in five appendices.

\section{The Dynamical Equations for Nutation and the Induction Equation for the Magnetic Field}

[11] Let the Earth be composed of a solid inner core, a fluid outer core, and an elastic mantle. In a first approximation, the Earth can be idealized as having a rheology satisfying an isotropic linearly elastic constitutive relation, and having an ellipsoidal shape consistent with hydrostatic equilibrium (HSE) in a steadily rotating reference frame. This ellipsoidal shape arises from the uniform rotation around the $z$ axis, with a constant angular speed $\Omega_{0}$, hydrostatic prestresses (stress tensor $\mathbf{T}^{\text {ref. }}$ ), and self-gravitation. In this case, the equal density and equipotential surfaces coincide, are axially symmetric and slightly ellipsoidal.

[12] Let $V_{E}$ be the volume occupied by the Earth at equilibrium, and consider that the origin and $z$ axis of the (uniformly rotating) reference frame attached to $V_{E}$ coincide with the geocenter and the rotation axis, respectively, and that the $x$ and $y$ axes rotate rigidly about the $z$ axis with a constant angular velocity $\Omega_{0}$ in space. At equilibrium, the Earth is in a HSE state and coincides exactly with $V_{E}$. When the equilibrium Earth is subjected to an infinitesimal timedependent disturbance, an additional displacement will be produced, with an additional stress tensor, and an additional gravitational potential due to the mass redistribution, which 


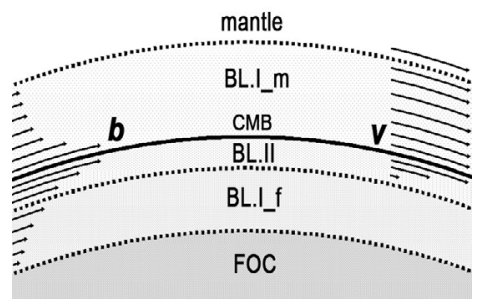

Figure 1. Schematic of the induced magnetic field $\mathbf{b}$ and the nutational velocity field $\mathbf{v}$ near the boundary. We take the case of $\mathrm{CMB}$ as an example and consider a rotating reference frame fixed to the FOC. Across the CMB, b should be continuous, while $\mathbf{v}$ is also continuous if it is nonslip boundary due to viscosity. At the fluid side, $\mathrm{v}$ varies rapidly over the radius of the very thin viscous layer (BL.II) and vanishes far away from the boundary, and the case of $\mathbf{b}$ is analogous to $\mathbf{v}$. At the solid side, $\mathbf{v}$ almost does not change over the radius of the thin conducting layer (BL.I_m), but $\mathbf{b}$ should be confined to this layer (and BL.I_f) and vanishes away from the boundary as in the fluid side.

all vary with time. The total stress tensor at each point inside the Earth then equals the sum of the reference stress and the additional stress tensor caused by the disturbance.

[13] Let $P$ be a particle in $V_{E}$ in HSE, and $\mathbf{r}$ its position vector. The equilibrium density field and the gravitational potential field are denoted by $\rho(\mathbf{r})$ and $\phi(\mathbf{r})$, respectively, while $\mathbf{s}(\mathbf{r}, t)$ denotes the infinitesimal Lagrangian displacement vector of particle $P$ at time $t$.

[14] The dynamic equation for infinitesimal elastic gravitational motion for a rotating, slightly elliptical Earth is given as, in a steadily rotating reference frame with constant speed $\Omega_{0}$ (see Smith [1974] or Dahlen and Tromp [1998] for more information):

$$
\begin{aligned}
\rho D_{t}^{2} \mathbf{s}+2 \rho \boldsymbol{\Omega}_{0} \times D_{t} \mathbf{s}= & -\rho \boldsymbol{\Omega}_{0} \times\left(\boldsymbol{\Omega}_{0} \times \mathbf{s}\right)+\nabla \cdot \mathbf{T}^{e}-\nabla(\gamma \nabla \cdot \mathbf{s}) \\
& -\rho \nabla \phi_{1}-\rho \mathbf{s} \cdot \nabla \nabla \phi+\nabla \cdot\left[\gamma(\nabla \mathbf{s})^{T}\right], \quad(1)
\end{aligned}
$$

where $\gamma$ is the equilibrium pressure, and $\phi_{1}$ the incremental gravitational potential induced by the mass redistribution due to deformation. The stress tensor $\mathbf{T}^{e}$ is the incremental stress with respect to the reference stress, $\mathbf{T}^{r e f .}=-\gamma \mathbf{I}$ where I is the identity tensor, and is related to the displacement field by two Lamé parameters $(\lambda, \mu)$ for an isotropic medium as

$$
\mathbf{T}^{e}=\lambda(\nabla \cdot \mathbf{s}) \mathbf{I}+\mu\left[\nabla \mathbf{s}+(\nabla \mathbf{s})^{T}\right],
$$

where rigidity $\mu=0$ in a liquid part of the Earth.

[15] Traditionally, when the magnetic contribution is neglected, the solution of equations (1) and (2) and Poisson's equation for a given Earth model (e.g., PREM) gives the displacement field $\mathbf{s}$ (and velocity field $\mathbf{v}=\partial_{t} \mathbf{s} \rightarrow i \omega \mathbf{s}$ ) everywhere in the interior, and the nutation is obtained from the toroidal part of degree 1 and order 1 of $\mathbf{s}$, denoted as $W_{1}^{1}$ (see later).

[16] Here, a magnetic field $\mathbf{B}_{0}$ which is stationary in time is added to the reference configuration. In the electrically conducting regions of the Earth $\mathbf{B}_{0}$ must be supported by a stationary electrical current system $\mathbf{j}_{0}=\left(\nabla \times \mathbf{B}_{0}\right) / \mu_{0}$. The electromagnetic field plus current can be stationary in time only if ohmic decay is offset by regenerative dynamo action, which in turn requires a flow field of velocity $\mathbf{v}_{0}$ (relative to the steadily rotating reference frame) in the FOC. (Another argument is that the magnetic field and its current can be assumed time invariant if their timescales are much longer than those of nutation to be discussed here.) Of course this nonzero velocity field will, for consistency, also have to be stationary in time. We are therefore assuming that we can establish an equilibrium configuration which is no longer one of hydrostatic equilibrium. To do this, we should show that we can obtain a consistent time-independent solution of the coupled system of differential equations: the momentum equation (with a Lorentz force term $\mathbf{j}_{0} \times \mathbf{B}_{0}$ per unit volume), the induction equation (with a term $\nabla \times\left(\mathbf{v}_{0} \times \mathbf{B}_{0}\right)$ ), and of course the solenoidal condition $\nabla \cdot \mathbf{B}_{0}=0$. In other words to be totally consistent physically we have to solve a stationary but nonlinear hydromagnetic dynamo problem! This is, however, a too difficult problem to solve, and we will just assume that adding a magnetic field still leaves us free to take the reference configuration as the hydrostatic equilibrium, wherever we need to do so. For the discussion of likely mechanisms to offset a finite background magnetic torque from the magnetic field and current, readers are recommended to refer related MHD studies.

[17] A small-oscillation departure from the reference configuration, such as nutation, is assumed and associated with a nonzero displacement field $\mathbf{s}$ (or velocity field $\mathbf{v}$ ) and a simultaneous perturbation $\mathbf{b}$ in the magnetic field. Of course both the nutation and the other components of $\mathbf{s}$ are modified by the presence of $\mathbf{b}$, and at the same time $\mathbf{b}$ depends on $\mathbf{v}$. So we have a differential system (the momentum equation and the induction equation) coupled by the Lorentz force and the induction term, to solve for $\mathbf{s}$ and $\mathbf{b}$ simultaneously.

[18] For computing EMC, we also assume, as shown in Figure 1, that there exist thin boundary layers, BL.I_f and BL.I_m, at both fluid and mantle sides of the CMB. They are magnetic skin layers and are supposed to be highly conducting although the bulk of the mantle is usually regarded as almost insulated. The magnetic field $\mathbf{b}$ induced by the nutation is assumed to act only within these layers, meaning that the coupling between $\mathbf{s}$ and $\mathbf{b}$ happens only within these boundary layers. The thickness of these two layers, i.e., the thickness of the magnetic boundary layer $\delta$ (it is named as "the skin layer depth" in paper 1 and paper 2), depends on the magnetic diffusivity $\eta$, on the magnetic model and on how the magnetic field varies in the layer. If a magnetic field is assumed to be reduced with the distance from the boundary by an exponential form, then $\delta=\sqrt{2 \eta /|\omega|}$. For a magnetic conductivity $\sigma_{f}=\sigma_{m}=5 * 10^{5} \mathrm{~S} / \mathrm{m}$ and the diurnal nutational frequency (1 day), we have $\delta_{f}=\delta_{m} \approx 200 \mathrm{~m}$.

[19] In traditional nutation studies, the outer core is considered as a pure liquid, meaning no viscosity and a free-slip boundary condition at the CMB. The mantle and the FOC (as well as the SIC) rotate rigidly with two different nutational angular velocities and there is a jump of velocity field in crossing the CMB. A very thin boundary layer (BL.II) in the fluid side is assumed, in which the rotational tangential velocities vary rapidly, and a large gradient in the direction normal to the boundary is expected in this very thin layer, 
while far away from the boundaries, the velocity field of the bulk of the FOC is still mainly the global nutational velocity induced by the external forcing. A possible candidate for this mechanism is viscosity as considered in some studies. On experimental grounds, the fluid in the core is of course not ideal and adherence to the boundary always takes place even if the viscosity is extremely small, and there is always a thin layer inside which the relative velocity decrease up to zero at the boundary. The thickness of this layer depends on the viscosity and is usually considered as very small (even smaller than $1 \mathrm{~m}$ ). Nevertheless, whether the viscosity of the fluid (or a combination of viscosity and magnetic field) is enough or is not enough to support the radial gradient of the tangential nutational velocity required as in paper 1 and paper 2 or in the work of Mathews and Guo [2005], is still uncertain and is an interesting question, and it will be discussed in a next paper. We just assume here that there is such a very thin layer in which there is a large radial gradient of the global nutational velocity. Although the CMB becomes nonslip at the sharp boundary and the velocity field should be continuous now, we still ignore in this work the hydrodynamic effect of this very thin viscous layer and as a result, there is still a jump (or discontinuity) of the nutational tangential velocity field if moving from the boundary point between the BL II and the bulk of the FOC to the CMB. We also do not consider the possible viscous force in the motion equation, and we only change the boundary condition at the $\mathrm{CMB}$ for the stress field in which the magnetic stress will take part (see section 2.3). Appendix A and Melchior [1986] also provide some information on the EMC and related fields near the boundary.

[20] The additional term in the equation of motion induced by the Lorentz force, $\mathbf{L}$, is added to the right hand side of equation (1) in both the core and the mantle near the CMB (and equivalently in both the inner core and the outer core near the ICB):

$$
\begin{aligned}
\rho D_{t}^{2} \mathbf{s}+2 \rho \boldsymbol{\Omega}_{0} \times D_{t} \mathbf{s}= & -\rho \boldsymbol{\Omega}_{0} \times\left(\boldsymbol{\Omega}_{0} \times \mathbf{s}\right)+\nabla \cdot \mathbf{T}^{e}-\nabla(\gamma \nabla \cdot \mathbf{s}) \\
& -\rho \nabla \phi_{1}-\rho \mathbf{s} \cdot \nabla \nabla \phi+\nabla \cdot\left[\gamma(\nabla \mathbf{s})^{T}\right]+\mathbf{L} .
\end{aligned}
$$

Note that this equation degenerates in the fluid core because the rigidity $\mu \rightarrow 0$ which will be discussed later.

[21] In principle, $\mathbf{L}$ may be exerted not only near the CMB and ICB but also throughout the Earth. However, we consider the Lorentz force to be efficient only in the thin layers near the CMB as mentioned above. The Lorentz force is indeed proportional to the radial derivative of the induced field, $\partial_{r} \mathbf{b}$, which vanishes away from the boundaries as $\mathbf{b}$ itself. This means that, when integrating the motion equation to compute the nutation from $\mathbf{s}$, we can still use the motion equation (1) everywhere in the Earth interior except in the thin boundary layers where the motion equation (3) applies.

\subsection{Approximation for $L$}

[22] The total magnetic force density can be expressed as

$$
\mathbf{L}^{\text {total }}=\nabla \cdot \mathbf{M}^{\text {total }},
$$

where $\mathbf{M}^{\text {total }}$ is the Maxwell magnetic stress tensor [Rochester, 1962]

$$
\mathbf{M}^{\text {total }}=\left(\mathbf{B B}-\frac{B^{2}}{2} \mathbf{I}\right) / \mu_{0} .
$$

Note that $\mu_{0}$ designates the magnetic permeability, rather than the rigidity $\mu$ in equation (2).

[23] Let total magnetic field $\mathbf{B}$ be decomposed into

$$
\mathbf{B}=\mathbf{B}_{0}+\mathbf{b},
$$

where $\mathbf{B}_{0}$ and $\mathbf{b}$ are the reference magnetic field and the magnetic field induced by nutation, respectively. We have $|\mathbf{b}| \ll\left|\mathbf{B}_{0}\right|$, and both are subject to the solenoidal condition $\nabla \cdot \mathbf{B}_{0}=\nabla \cdot \mathbf{b}=0$. Then

$$
\mathbf{M}^{\text {total }}=\left(\mathbf{B}_{0} \mathbf{B}_{0}+\mathbf{B}_{0} \mathbf{b}+\mathbf{b} \mathbf{B}_{0}+\mathbf{b b}-B^{2} \mathbf{I} / 2\right) / \mu_{0}
$$

[24] In all the following text including appendixes, we are interested only in the diurnal frequency band in which nutations lie.

[25] The reference magnetic field $\mathbf{B}_{0}$ (that does not vary at diurnal timescale in an Earth-fixed reference frame) and its magnetic stress $\left(\mathbf{B}_{0} \mathbf{B}_{0}\right)$ can be considered as a part of the reference state in the absence of any nutational motion. Meanwhile, the dynamical effect of the term $B^{2} \mathbf{I} / 2$ (a magnetic pressure) on nutation is indistinguishable from that of the mechanical pressure and is absorbed into the effective pressure [Greenspan, 1968]. Therefore, when considering the contribution of the EMC to nutation, we reduce the magnetic stress tensor to

$$
\mathbf{M}=\left(\mathbf{B}_{0} \mathbf{b}+\mathbf{b} \mathbf{B}_{0}+\mathbf{b b}\right) / \mu_{0}
$$

[26] Generally, the induced field is much smaller than the main field (b $\ll \mathbf{B}_{0}$ ). Therefore, bb can be ignored in comparison with the other terms (it would also induce components outside of the diurnal band), i.e.,

$$
\mathbf{M}=\left(\mathbf{B}_{0} \mathbf{b}+\mathbf{b B}_{0}\right) / \mu_{0},
$$

and the terms in $\mathbf{L}$ that must be kept in equation (3) for nutation are

$$
\mathbf{L} \approx\left(\mathbf{B}_{0} \cdot \nabla \mathbf{b}+\mathbf{b} \cdot \nabla \mathbf{B}_{0}\right) / \mu_{0}
$$

[27] As the incremental magnetic field $\mathbf{b}$ is confined to the thin boundary layer, its gradient is important. The scale of $\mathbf{B}_{0} \cdot \nabla \mathbf{b}$ is $B_{0} b / \delta_{b}$, while that of $\mathbf{b} \cdot \nabla \mathbf{B}_{0}$ is $B_{0} b / L_{B 0}$, where $\delta_{b} \ll L_{B 0}$, so the second term can be ignored compared with the first one. We then have

$$
\mathbf{L} \approx\left(\mathbf{B}_{0} \cdot \nabla \mathbf{b}\right) / \mu_{0}
$$

[28] The Lorentz force can be decomposed into a sum of two parts, one related to the poloidal part of main field $\mathbf{B}$, the other to the toroidal component of the main field. Concerning the toroidal part of the reference magnetic field, $\mathbf{B}_{0}^{T}(\mathbf{r})$, it is natural for a perfectly conducting FOC to have a 
toroidal magnetic field. However, it is not observable at the Earth's surface and the only way to obtain the features of $\mathbf{B}_{0}^{T}(\mathbf{r})$ is from geodynamo models, which, while already complicated to run, are mostly far simpler than in reality. One can, anyway, use numerical models of $\mathbf{B}_{0}^{T}(\mathbf{r})$ (coefficients of spherical harmonics) from geodynamo models [e.g., Kuang and Bloxham, 1999] (see also Glatzmaier [2002] or Christensen and Wicht [2007] for a complete review), or from an approximate relationship between $\mathbf{B}_{0}^{T}(\mathbf{r})$ and $\mathbf{B}_{0}^{r}(\mathbf{r})$. For example, based on a geostrophic flow model, Stix and Roberts [1984] give the following relationship for a conducting shell in which conductivity $\sigma_{f}(r)$ decreases rapidly with radius:

$$
\mathbf{B}_{0}^{T} \approx-B_{0}^{r}\left[h / \eta_{a_{c}}\right] \mathbf{u}_{a_{c}},
$$

where $\mathbf{u}_{a}$ is the geostrophic flow velocity at the CMB with mean radius $a_{c}$ and has mainly longitudinal (along $\hat{\varphi}$ ) components, $\eta_{a_{c}}$ is the magnetic diffusivity at the CMB, and $h$ is the length scale over which the shell conductivity varies. For typical $\mathbf{B}_{0}^{T}$ values from geodynamo models, Buffett [1993] has shown that the toroidal contribution to nutation is most likely not large enough to be observable.

[29] Moreover, the horizontal derivatives of $\mathbf{b}$ are further assumed to be negligible in comparison with the radial derivative, $\nabla_{H} \mathbf{b} \ll \partial_{r} \mathbf{b}$ (see Appendix A). The magnetic force is then approximated as

$$
\mathbf{L} \approx \frac{1}{\mu_{0}} B_{0}^{r} \partial_{r} \mathbf{b}
$$

and depends on $B_{0}^{r}$, the radial component of $\mathbf{B}_{0}$, and on $\partial_{r} \mathbf{b}$ at the CMB, where $B_{0}^{r}$ can be obtained from downward continuation from the observed values at the outer surface to the $\mathrm{CMB}$, and $\partial_{r} \mathbf{b}$ is obtained from solving the induction equation as below.

\subsection{On the Induction Equation and Its Solution}

[30] Generally, the induction equation is [Moffatt, 1978]

$$
\partial_{t} \mathbf{B}=\nabla \times\left[\left(\mathbf{v}_{0}+\mathbf{v}\right) \times \mathbf{B}\right]-\nabla \times(\eta \nabla \times \mathbf{B}),
$$

where, both $\mathbf{v}_{0}$, the reference velocity field in the core supporting the reference magnetic field for its long-term component, and the nutational velocity $\mathbf{v}$ in the diurnal band are involved.

[31] With the approximations explained in Appendix A, the perturbation of the induction equation due to nutation can be written up to the first order in the all quantities as

$$
\partial_{t} \mathbf{b} \approx \nabla \times\left(\mathbf{v} \times \mathbf{B}_{0}\right)+\eta \nabla^{2} \mathbf{b} \approx B_{0}^{r} \partial_{r} \mathbf{v}+\eta \nabla^{2} \mathbf{b} .
$$

In this approximated induction equation, the induced magnetic field $\mathbf{b}$ depends on $B_{0}^{r}$ and $\mathbf{v}$ only, while $\mathbf{v}_{0}$ is not involved anymore.

[32] In principle, one can use this equation and integrate it together with the equation of motion to solve $\mathbf{b}$ as well as $\mathbf{s}$ (or v). Starting from here, Huang et al. [2005] expanded the vector equation (15) to scalar equations in generalized surface spherical harmonics (GSSH) along with the equations of motion, which provide the theoretical basis for solving simultaneously the nutation and the perturbed magnetic field.
As a first step, in this paper we will try to verify the results obtained by the MHB model. Therefore, we will use the same hypotheses as in that paper and base our computation on the approximations used in paper 1 and later in paper 2 . The difference between paper 1 and paper 2 will be discussed later.

[33] Because of all the hypotheses considered, $\mathbf{b}$ and $\mathbf{v}$ have only tangential components (i.e., $b_{r}=v_{r}=0$ ) in the very thin boundary layer at the CMB $\left(r=a_{c}\right)$. There are two boundary conditions on $\mathbf{b}$. The first is that $\mathbf{b}$ be continuous across $\mathrm{CMB}$, and the second is that

$$
\left[\eta \partial_{r} \mathbf{b}\right]_{-}^{+}=\eta_{m}\left(\partial_{r} \mathbf{b}\right)_{m}-\eta_{f}\left(\partial_{r} \mathbf{b}\right)_{f}=B_{0}^{r} \mathbf{v}_{f-m} .
$$

The latter can be obtained by integrating the induction equation (15) over radius in the thin boundary layer across the velocity discontinuity at $\mathrm{CMB}$ and by noting that the integration of $\partial_{r} \mathbf{v}$ across the boundary is just $\mathbf{v}_{f-m}$, where $\mathbf{v}_{f-m}$ is the nutational velocity of the fluid relative to the mantle, i.e.,

$$
\mathbf{v}_{f-m}=\mathbf{v}_{f o c}^{c m b}-\mathbf{v}_{\text {mantle }}^{c m b} \approx\left(\boldsymbol{\Omega}_{f}-\boldsymbol{\Omega}_{m}\right) \times \mathbf{r},
$$

in which $\boldsymbol{\Omega}_{f}$ and $\boldsymbol{\Omega}_{m}$ are the total angular velocity vectors of the FOC and the mantle, respectively, and subscripts $(m, f)$ denote the mantle and FOC side, respectively. In what follows we will consider only the mantle for the solid part; the case near the SIC can be obtained in a similar manner.

[34] In the first case, we assume that the conductivity profile in the thin layer BL.I_m decays away from the CMB with the following exponential form

$$
\sigma(r)=\sigma_{c m b} e^{-\left(r-a_{c}\right) / \Delta},
$$

where $\sigma_{c m b}$ is the mantle conductivity at the CMB and $\Delta$ is the characteristic thickness of the conducting layer BL.I_m. By using the above two boundary conditions on $\mathbf{b}$, it is not difficult to get the solutions in the thin layers BL.I $f$ and BL.I_m as (see paper 1 for detail)

$$
\partial_{r} \mathbf{b}(r)=C_{1} \mathbf{b}(r)
$$

and the induced magnetic field $\mathbf{b}$ at $\mathrm{CMB}\left(r=a_{c}\right)$

$$
\mathbf{b}\left(a_{c}\right) \equiv\left(b_{\theta} \hat{\theta}+b_{\varphi} \hat{\varphi}\right)=-k_{1} B_{0}^{r} \mathbf{v}_{\mathbf{f}-\mathbf{m}},
$$

where

$$
C_{1}= \begin{cases}(1+i) / \delta_{f} & \text { in BL.I_f } \\ -\frac{J_{0}\left(z e^{-i \pi / 4}\right)}{J_{1}\left(z e^{-i \pi / 4}\right)} \frac{z}{2 \Delta} e^{-i \pi / 4} & \text { in BL.I_m }\end{cases}
$$

and

$$
k_{1}=\frac{e^{i \pi / 4}}{\sqrt{2}} \frac{J_{1}\left(z_{0} e^{-i \pi / 4}\right)}{\frac{\eta_{m}}{\delta_{m}} J_{0}\left(z_{0} e^{-i \pi / 4}\right)+i \frac{\eta_{f}}{\delta_{f}} J_{1}\left(z_{0} e^{-i \pi / 4}\right)}
$$

is a function of $z_{0}$, in which, $J_{n}$ is the $n$th degree Bessel function of the first kind; the parameter $z$ in equation (21) is defined as $z=\sqrt{8}\left(\frac{\Delta}{\delta_{m}}\right) e^{-\left(r-a_{c}\right) / 2 \Delta}$, and

$$
z_{0} \equiv z\left(a_{c}\right)=\sqrt{8} \Delta / \delta_{m},
$$


where $\delta_{m}$ is the boundary thickness in the mantle and may have the form [Gubbins and Roberts, 1987] $\delta_{m}=\sqrt{2 \eta_{m} / \omega}$. The characteristic thickness of the conducting layer $\Delta$ takes $200 \mathrm{~m}$ in this paper; taking $\sigma_{\mathrm{cmb}}$ as $5 * 10^{5} \mathrm{Sm}^{-1}$ and we have $\delta_{m} \approx 4.7 \mathrm{~km}$ for diurnal nutation.

[35] In the more simple second case, we assume that the conductivities in the two boundary layers are spatially uniform (and one can assume for further simplicity as in this paper for comparison, but not necessary, that $\delta_{f}=\delta_{m}$ ). The solutions in the thin boundary layers will be

$$
\partial_{r} \mathbf{b}(r)=C_{2} \mathbf{b}(r)
$$

and at $\mathrm{CMB}$

$$
\mathbf{b}\left(a_{c}\right)=-k_{2} B_{0}^{r} \mathbf{v}_{f-m},
$$

in which

$$
C_{2}=\left\{\begin{array}{l}
(1+i) / \delta_{f} \quad \text { in BL.I_f } \\
-(1+i) / \delta_{m} \quad \text { in BL.I_m }
\end{array}\right.
$$

and

$$
k_{2}=\frac{(1-i)}{2\left(\eta_{f} / \delta_{f}+\eta_{m} / \delta_{m}\right)}=\frac{(1-i)}{\sqrt{2 \eta_{m}|\omega|}+\sqrt{2 \eta_{f}|\omega|}},
$$

the coefficient $k_{2}$ depends on the diffusivity $(\eta)$ and the thickness $(\delta)$ of the magnetic boundary layers in the vicinity of the CMB.

[36] Once again, $\partial_{r} \mathbf{b}$ is not continuous across CMB as the $C_{1}$ and $C_{2}$ are different for the two sides.

[37] As the third case, in paper 2 where similar approximations are used but the Coriolis force is included, the induced magnetic field has the following form in the boundary layers

$$
\begin{aligned}
b_{ \pm}(\mathbf{r}, t) \equiv & b_{\theta} \pm i b_{\varphi} \\
= & b_{ \pm}^{(+)}(r)(1 \pm \cos \theta) e^{i(\omega t-\varphi)} \\
& +b_{ \pm}^{(-)}(r)(1 \mp \cos \theta) e^{-i(\omega t-\varphi)}
\end{aligned}
$$

where the coefficients $b_{ \pm}^{(+)}(r)=b_{ \pm}^{(+)}\left(a_{s}\right) e^{\lambda_{f \pm}\left(r-a_{s}\right)}$ with the subscript $s$ denoting the mantle $\left(a_{s}=a_{c}\right.$ at $\left.\mathrm{CMB}\right)$ or SIC $\left(a_{s}=a_{I C B}\right.$ at ICB). At the boundaries

$$
b_{ \pm}^{(+)}\left(a_{s}\right)=k_{3} B_{0}^{r} v_{s-f}\left(a_{s}\right)
$$

where

$$
k_{3}=\frac{1}{\left(i \omega / \lambda_{f \pm}\right)-\eta_{s} \lambda_{s}},
$$

and $v_{s-f}\left(a_{s}\right)=i a_{s} \Delta \omega_{s-f} / 2$ (where the factor $1 / 2$ is introduced by the definition of $\mathbf{b}$ in canonical form in equations (29) and (28)) and $\Delta \omega_{s-f}$ are the velocity and the angular velocity, respectively, of the solid part (mantle or SIC) with respect to the FOC. And $b_{ \pm}^{(-)}$is equal to the conjugate of $b_{ \pm}^{(+)}$.

[38] The other parameters used in equation (30) are the spatial oscillation frequency of the induced magnetic field at fluid $\left(\lambda_{f}\right)$ and solid $\left(\lambda_{s}\right)$ sides near the boundaries:

$$
\lambda_{f \pm}^{2}=\frac{-\omega(\omega \pm f)}{\left(B_{0}^{r}\right)^{2} /\left(\mu_{0} \rho\right)+i \eta_{f}(\omega \pm f)}, \quad \lambda_{s}^{2}=\frac{i \omega}{\eta_{s}},
$$

where $f=2 \Omega_{0} \cos \theta$ and $\omega$ is the nutation frequency. The signs of $\lambda_{f}$ and $\lambda_{s}$ are imposed by the boundary condition and the definition of the outward unit vector of the boundary sphere, i.e.,

$$
\operatorname{Re}\left(\lambda_{f+}\right)>0, \quad \operatorname{Re}\left(\lambda_{s+}\right)<0
$$

for the CMB. Implicitly, the quantities above are in the frequency domain, meaning, for example, $b_{ \pm}^{(+)}(r)=b_{ \pm}^{(+)}(r, \omega)$.

[39] From the solution form above, one can easily see that b has a linear dependence on $B_{0}^{r}$ and has no radial part (i.e., $\mathbf{b}$ is horizontal), and that $\mathbf{b}$ is parallel to and only related to the relative velocities $\mathbf{v}_{S^{-} f}$ of the mantle (or SIC) with respect to the liquid core at the first order.

[40] Comparing the three solution forms, equations (19)(22), equations (24)-(27) and equations (28)-(31), one can find that all solutions of $\mathbf{b}(r)$ have the same exponential form with radial coordinate $r$ and that the boundary thickness $\delta$ relates to the spatial frequency $\lambda$ by $\lambda_{s}=(1+i) / \delta_{s}$ and analogous for $\delta_{f}$ and $\lambda_{f}$ if ignoring $\left(B_{0}^{r}\right)^{2} / \mu_{0} \rho$ and $f$ in $\lambda_{f}$.

[41] As the boundary layers are believed to be so thin, the two solutions given by paper 1 and paper 2 are physically equivalent, except for two contributions that can be seen in equations (30) and (31): (1) a small contribution from the Coriolis force in the fluid and (2) the influence of the magnetic field on the spatial oscillation frequency.

[42] In section 3.2 we will verify that when taking $\left(B_{0}^{r}\right)^{2} / \mu_{0} \rho$ and $f$ out of $\lambda_{f}$ in equation (31), one obtains the same expressions for $k$ in equation (30) as in equation (27).

\subsection{Incorporating $L$ Into the Scalar ODE}

[43] We consider in this section the second solution of $\mathbf{b}$ (equations (24)-(27)), and a discussion of the other two solutions will be given in section 3. Taking the $\mathrm{CMB}$ as an example (the case at the ICB is analogous), we have

$$
\partial_{r} \mathbf{b}=-C_{2} k_{2} B_{0}^{r} \mathbf{v}_{f-m} .
$$

Therefore, the Lorentz force density becomes

$$
\mathbf{L} \approx-\frac{1}{\mu_{0}} C_{2} k_{2}\left(B_{0}^{r}\right)^{2} \mathbf{v}_{f-m}
$$

or transformed into nutational frequency domain as

$$
\mathbf{L} \approx K \mathbf{s}_{f-m}
$$

where

$$
K=-\frac{i \omega}{\mu_{0}} C_{2} k_{2}\left(B_{0}^{r}\right)^{2}
$$

where $i \omega$ transfers the parameters from time domain to nutational frequency domain. The $\omega$ can be any frequency as it is just a general Fourier transform here, but in this paper, it can be regarded as the nutation frequency only. Please notice that $K$ is different at the two sides of $\mathrm{CMB}$ as is $C_{2}$.

[44] Because the relative velocity field $\mathbf{v}_{f-m}$ considered here and in equation (17) is only the global rigid rotation of the whole layer at the FOC side with respective to the whole layer at the mantle side, similarly to Smith [1974], the part in 
the displacement field $\mathbf{s}$ to be kept here is only the toroidal part $\tau$ of degree $l=1$ and order $m=1$, i.e.,

$$
\tau_{1}^{1}(\mathbf{r}, \omega)=\frac{1}{2} W_{1}^{1}(r, \omega)\left[-\hat{e}_{-} D_{1-}^{1}(\theta, \varphi)+\hat{e}_{+} D_{1+}^{1}(\theta, \varphi)\right] .
$$

[45] $W_{l}^{m}$, and $U_{l}^{m}$ and $V_{l}^{m}$ that will appear later, are the toroidal, radial and transverse spheroidal components, respectively, of the displacement $\mathbf{s}$ of degree $l$ and order $m$; and $D_{m \alpha}^{l}(\theta, \varphi)$ is the GSSH defined by Smith [1974]. The above equation in which only the term of degree 1 and order 1 is kept implies that only the rigid rotation angle is considered and that the deviations from rigid-body motion (i.e., the contribution from $U_{2}^{1}, V_{2}^{1}$ and others) are ignored. This approximation for the velocity (and displacement) field is consistent with other approximations made in our discussion of the EMC.

[46] It can be seen from equations (35) and (37) that $\mathbf{L}$ is toroidal. Because $\mathbf{L}$ is added to the stress term in the equation of motion to include the magnetic force, all we need to change is just to replace the toroidal part of the stress term $\partial_{r} R_{1}^{1}$ by $\partial_{r} R_{1}^{1}+\left(\partial_{r} R_{1}^{1}\right)^{\text {incr. }}$ in the equation of motion containing $\partial_{r} R_{1}^{1}$ [i.e., Smith, 1974, equation (5.30)], for both the mantle side and the fluid side, where, $R_{l}^{m}, Q_{l}^{m}$ and $P_{l}^{m}$ that will appear later are the toroidal, transverse spheroidal and radial components, respectively, of the stress $\mathbf{T}$ of degree $l$ and order $m .\left(\partial_{r} R_{1}^{1}\right)^{i n c r}$. is the incremental part of $\partial_{r} R_{1}^{1}$ due to EMC. This replacement is as follows:

$$
\partial_{r} R_{1}^{1} \rightarrow \partial_{r} R_{1}^{1}+\left(\partial_{r} R_{1}^{1}\right)^{i n c r},
$$

where

$$
\left(\partial_{r} R_{1}^{1}\right)^{\text {incr. }}=K\left[W_{1}^{1}\right]_{f-m},
$$

and $\left[W_{1}^{1}\right]_{f-m}=\left[W_{1}^{1}\right]_{f}-\left[W_{1}^{1}\right]_{m}$ at the CMB, which can be obtained after integration for each nutation frequency $\omega$. This is discussed in detail in section 2.4.

[47] Let us now return to the equation of motion (3). b is confined to the boundary layers, so equation (3) with expression (34) for the magnetic force must only be considered in the thin boundary layers. It must be noted that equations (2) and (3) are degenerate for the fluid outer core, i.e., the equations governing the radial derivatives of the tangential displacement field that are derived from equation (2) and depend on $1 / \mu$ [see, e.g., Smith, 1974, equations (5.23) and (5.24)] are not valid for fluid regions as the Lamé parameter $\mu=0$ for an ideal fluid. Instead, one can derive them from the equation of motion by setting the tangential stress and their radial derivatives equal to zero (for details, see Huang et al. [2004] for the case without magnetic field). This results in the following format:

$$
\mathbf{D} \cdot \tilde{\mathbf{X}}=\mathbf{E} \cdot \tilde{\mathbf{Y}}
$$

where

$$
\begin{gathered}
\tilde{\mathbf{X}} \equiv \tilde{\mathbf{X}}(r, \omega)=\left\{V_{l}^{m}(r, \omega), W_{l-1}^{m}(r, \omega), W_{l+1}^{m}(r, \omega)\right\}^{T}, \\
\tilde{\mathbf{Y}} \equiv \tilde{\mathbf{Y}}(r, \omega)=\left\{U_{l}^{m}(r, \omega), P_{l}^{m}(r, \omega), \phi_{1 l}^{m}(r, \omega), g_{1 l}^{m}(r, \omega)\right\}^{T}
\end{gathered}
$$

are the sets of scalars for the tangential displacement and for all nonvanishing spheroidal variables, respectively, except $V_{l}^{m}$; and $\mathbf{D}$ and $\mathbf{E}$ are $3 \times 3$ and $3 \times 4$ coefficient matrices that only depend on the degree $(l)$ and order $(m)$ of the external potential, the density $(\rho)$, the Lamé parameters $(\lambda, \mu)$, the radius $(r)$, the frequency $(\omega)$, the equilibrium gravitational potential $\left(\phi_{0}, \phi_{2}\right)$, and the equilibrium gravity $\left(g, \tilde{g}, \partial_{r} \phi_{2}\right)$, all of which can be obtained from a given Earth model like PREM; $\phi_{1 l}^{m}$ is the incremental Eulerian gravitational potential due to displacement, and $g_{1 l}^{m}$ is the corresponding incremental gravitational flux defined by $g_{1 l}^{m}=\partial_{r} \phi_{1 l}^{m}+4 \pi G \rho_{0} U_{l}^{m}$.

[48] In summary, for the numerical integration method, the governing equations in the FOC form a set of four ODEs of the first order in $d / d r$ [i.e., Smith, 1974, equations (5.22), (5.25), (5.26), and (5.28)], which can be expressed as

$$
\partial_{r} \tilde{\mathbf{Y}}=\mathbf{A}_{1} \cdot \tilde{\mathbf{Y}}+\mathbf{A}_{2} \cdot \tilde{\mathbf{X}}+\mathbf{A}_{3} \cdot \partial_{r} \tilde{\mathbf{X}}
$$

where $\tilde{\mathbf{X}}$ can be calculated from the known $\tilde{\mathbf{Y}}$ by equation (40) and $\partial_{r} \tilde{\mathbf{X}}$ can also be derived from equation (40) by taking a radial derivative [see Huang et al., 2004]:

$$
\mathbf{D} \cdot \partial_{r} \tilde{\mathbf{X}}=\partial_{r}[\mathbf{E} \cdot \tilde{\mathbf{Y}}]-\left(\partial_{r} \mathbf{D}\right) \cdot \tilde{\mathbf{X}}
$$

[49] When introducing the Lorentz force for the FOC in the equations above, the three scalar equations (40) need to be changed to

$$
\mathbf{D} \cdot \tilde{\mathbf{X}}=\mathbf{E} \cdot \tilde{\mathbf{Y}}+K_{f} \tilde{\mathbf{X}}_{f-m}
$$

This is related to the changes in the equation corresponding to setting $\partial_{r} R_{1}^{1}=0$ in equation (5.30) of Smith [1974] by considering

$$
\partial_{r} R_{1}^{1}=0+\left(\partial_{r} R_{1}^{1}\right)^{i n c r .}=K_{f}\left[W_{1}^{1}\right]_{f-m},
$$

where $K_{f}$ is the value of $K$ at the fluid side.

\subsection{On the Boundary Condition at CMB}

[50] In addition to the change in the motion equation for both mantle and fluid sides, one also needs to modify the boundary conditions on the stress field at the CMB $\left(r=a_{c}\right)$.

[51] Traditionally, the boundary condition on stress field $\mathbf{T}^{e}$ at CMB (as well as at all kind of boundaries) is that $\hat{\mathbf{n}}(\mathbf{p}) \cdot$ $\mathbf{T}^{e}(\mathbf{p})$ be continuous across the boundary, i.e.,

$$
\hat{\mathbf{n}}(\mathbf{p}) \cdot \mathbf{T}^{e}(\mathbf{p}) \quad \text { cont. },
$$

where

$$
\mathbf{p}=\mathbf{r}+\hat{\mathbf{r}} h=\hat{\mathbf{r}}(r+h)
$$

is the material point on the boundary in the bulk volume $\mathscr{V}_{E}$ and $\mathbf{r}$ is its corresponding point in the effective spherical domain (ESD). The normal to the boundary can be expressed as

$$
\hat{\mathbf{n}}(\mathbf{p})=\hat{\mathbf{r}}\left(1+\partial_{r} h\right)-\nabla h=\hat{\mathbf{r}}+\hat{\theta} \frac{h_{2}}{r} P_{2}^{1}(\cos \theta),
$$


where all are expressed in the ESD and

$$
h(r, \theta)=h_{2}(r) P_{2}(\cos \theta)=-\frac{2}{3} r \epsilon(r) P_{2}(\cos \theta) .
$$

Expanding also $\mathbf{T}^{e}(\mathbf{p})=\mathbf{T}^{e}(\mathbf{r}+\hat{\mathbf{r}} h)$ in a Taylor series, then the boundary condition on stress field (equation (45)) becomes, expressed in the ESD of radius $r$ and to first order in ellipticity, as

$$
\hat{\mathbf{r}} \cdot \mathbf{T}^{e}+h \hat{\mathbf{r}} \cdot \partial_{r} \mathbf{T}^{e}+\mathbf{T}^{e} \cdot\left(\hat{\mathbf{r}} \partial_{r} h-\nabla h\right) .
$$

[52] If the Maxwell stress $\mathbf{M}$ is incorporated, the new boundary condition on the stress field at the CMB becomes

$$
\hat{\mathbf{n}}(\mathbf{p}) \cdot\left(\mathbf{T}^{e}(\mathbf{p})+\mathbf{M}(\mathbf{p})\right) \quad \text { cont } .
$$

Following section 2.1 and focusing only on the diurnal frequency band in which nutations lie, $\mathbf{M}$ takes the form of equation (9). Substituting it into the new boundary condition equation (50), and expanding them to ESD as done for the dynamical stress $\mathbf{T}^{e}$, the continuity quantities in the new boundary condition then, additional to those in equation (49), include the following terms:

$$
\begin{aligned}
\hat{\mathbf{r}} \cdot \mathbf{M} & +h \hat{\mathbf{r}} \cdot \partial_{r} \mathbf{M}+\mathbf{M} \cdot\left(\hat{\mathbf{r}} \partial_{r} h-\nabla h\right) \\
= & \frac{1}{\mu_{0}}\left[B_{0}^{r} \mathbf{b}+b^{r} \mathbf{B}_{0}+h\left(\partial_{r} B_{0}^{r} \mathbf{b}+B_{0}^{r} \partial_{r} \mathbf{b}+\partial_{r} b^{r} \mathbf{B}_{0}+b^{r} \partial_{r} \mathbf{B}_{0}\right)\right. \\
& \left.+\left(\mathbf{B}_{0} b^{\theta}+\mathbf{b} B_{0}^{\theta}\right) \frac{h_{2}}{r} P_{2}^{1}\right],
\end{aligned}
$$

where all the above quantities are expressed in ESD of radius $r$, and both the initial (reference) magnetic field $\mathbf{B}_{0}$ and the induced magnetic field $\mathbf{b}$ should be continuous across the CMB and ICB.

[53] Meanwhile, since the radial derivative of the radial component of $\mathbf{B}_{0}, \partial_{r} B_{0}^{r} \approx-\frac{l+2}{a_{c}} B_{0}^{r}$, is also continuous across the CMB. Moreover, according to the assumption that $\mathbf{b}$ has only tangential components (parallel to the differential velocity across the boundary $\mathbf{v}_{f-m}$ ) and does not have a radial component, we have $b^{r}=\partial_{r} b^{r}=0$. Therefore, additional to those in equation (49), there is only one term related to Maxwell stress kept in the new continuity quantity, i.e., $\frac{h}{\mu_{0}} B_{0}^{r} \partial_{r} \mathbf{b}$, where $\partial_{r} \mathbf{b}$ is not continuous. If assuming that the diffusivities at fluid side and at mantle side equal, i.e., $\eta_{f}=\eta_{m} \equiv \eta$, then from equation (16), one has

$$
\Delta\left(\partial_{r} \mathbf{b}\right)=\left[\partial_{r} \mathbf{b}\right]_{-}^{+}=1 / \eta B_{0}^{r} \mathbf{v}_{f-m}
$$

and the new boundary condition becomes

$$
\left[\hat{\mathbf{r}} \cdot \mathbf{T}^{e}+h \hat{\mathbf{r}} \cdot \partial_{r} \mathbf{T}^{e}+\mathbf{T}^{e} \cdot\left(\hat{\mathbf{r}} \partial_{r} h-\nabla h\right)\right]_{-}^{+}+\frac{h}{\eta \mu_{0}}\left(B_{0}^{r}\right)^{2} \mathbf{v}_{f-m}=0 .
$$

Using equation (48) and $\frac{1}{\eta \mu_{0}}=\sigma$ then

$$
\left[\hat{\mathbf{r}} \cdot \mathbf{T}^{e}+h \hat{\mathbf{r}} \cdot \partial_{r} \mathbf{T}^{e}+\mathbf{T}^{e} \cdot\left(\hat{\mathbf{r}} \partial_{r} h-\nabla h\right)\right]_{-}^{+}+h_{2} P_{2} \sigma\left(B_{0}^{r}\right)^{2} \mathbf{v}_{f-m}=0
$$

in time domain, or

$$
\hat{\mathbf{r}} \cdot \mathbf{T}^{e}+h \hat{\mathbf{r}} \cdot \partial_{r} \mathbf{T}^{e}+\mathbf{T}^{e} \cdot\left(\hat{\mathbf{r}} \partial_{r} h-\nabla h\right)+\kappa P_{2} \mathbf{s} \quad \text { cont. }
$$

in frequency domain, where $\mathbf{s}$ is displacement field $(\mathbf{v} \rightarrow i \omega \mathbf{s})$, $\kappa=-i \omega \sigma h_{2}\left(B_{0}^{r}\right)^{2}$, and $h_{2}=-(2 / 3) r \epsilon$.

[54] Noting that

$$
P_{2} \mathbf{s}=\sum_{\alpha} \hat{e}^{\alpha} \sum_{l, m}\left\{\sum_{l^{\prime}=|l-2|}^{l+2}\left[\begin{array}{ccc}
l & 2 & l^{\prime} \\
\alpha & 0 & \alpha \\
m & 0 & m
\end{array}\right] S_{l^{\prime}}^{m \alpha}\right\} D_{m \alpha}^{l}
$$

the new continuity quantities on the stress field components $(P, Q, R)$ are changed with additional terms $\left(P^{\text {incr. }}, Q^{\text {incr. }}, R^{\text {incr. }}\right)$ to equations (5.44)-(46) of Smith [1974] or equations (47)(49) of Huang et al. [2001]:

$$
\begin{aligned}
& \left(P_{l}^{m}\right)^{i n c r .}=\kappa \sum_{l^{\prime}=|l-2|}^{l+2},\left[\begin{array}{ccc}
l & 2 & l^{\prime} \\
0 & 0 & 0 \\
m & 0 & m
\end{array}\right] U_{l^{\prime}}^{m} \\
& \left(Q_{l}^{m}\right)^{\text {incr. }}=\kappa \sum_{l^{\prime}=|l-2|}^{l+2}\left[\begin{array}{ccc}
l & 2 & l^{\prime} \\
+ & 0 & + \\
m & 0 & m
\end{array}\right]\left[\begin{array}{c}
V_{l^{\prime}}^{m} \\
W_{l^{\prime}}^{m}
\end{array}\right] \begin{array}{c}
\left|l-l^{\prime}\right| \text { even } \\
\left|l-l^{\prime}\right| \text { odd }
\end{array}, \\
& \left(R_{l}^{m}\right)^{\text {incr. }}=\kappa \sum_{l^{\prime}=|l-2|}^{l+2}\left[\begin{array}{ccc}
l & 2 & l^{\prime} \\
+ & 0 & + \\
m & 0 & m
\end{array}\right]\left[\begin{array}{c}
W_{l^{\prime}}^{m} \\
V_{l^{\prime}}^{m}
\end{array}\right] \mid \begin{array}{l}
\left|l-l^{\prime}\right| \text { even } \\
\left|l-l^{\prime}\right| \text { odd }
\end{array}
\end{aligned}
$$

[55] In the frame of EMC and on the assumption in that frame that only the rigid nutational rotation between FOC and mantle is considered, we can then ignore the contribution from $\left(P_{l}^{m}\right)^{\text {incr. }}$ (equation (57)) and the contributions to both $\left(Q_{l}^{m}\right)^{i n c r .}$ and $\left(R_{l}^{m}\right)^{i n c r .}$ from $V_{l^{\prime}}^{m}$.

\subsection{Iteration: A Practical Strategy to Solve the Coupling ODE System}

[56] The nutation velocity $\mathbf{v}$ results, for a part, from both the reference magnetic field $\mathbf{B}_{0}$ and the incremental magnetic field $\mathbf{b}$, while $\mathbf{b}$ depends on the differential nutation velocity $\mathbf{v}_{f-m}$ at the boundaries; this is why nutation and magnetic field are coupled to each other. Without EMC, equations (40) and (41) can be solved together by substitution and solving for one set of variables $(\tilde{\mathbf{Y}}), \tilde{\mathbf{X}}$ can then be obtained from the known $\tilde{\mathbf{Y}}$ with equation (40), and one gets $\partial_{r} \tilde{\mathbf{X}}$ from equation (42). Introducing magnetic perturbation renders the above procedure no longer possible because of the coupling between the fluid and the mantle: the quantities $\tilde{\mathbf{X}}$ at fluid side depend on $\tilde{\mathbf{X}}$ at mantle side from equation (43), the latter one is determined from the solution of $(\tilde{\mathbf{Y}}, \tilde{\mathbf{X}})$ at fluid side and from the new boundary condition equation (50) or (55) in which the $\tilde{\mathbf{X}}$ at both the fluid and the mantle sides are involved.

[57] In order to solve this coupled system, we use an iteration strategy because the magnetic perturbation to the nutation is small compared to the nutation induced by the external forcing. We take the electromagnetic coupling at the $\mathrm{CMB}$ as an example. First, we integrate the equation of motion (1) without the perturbed magnetic field as usual throughout the Earth and obtain the mean global rotation, or $\left[W_{1}^{1}\right]_{f}$ and $\left[W_{1}^{1}\right]_{m}$. Secondly, from these values, we determine $\left(\partial_{r} R_{1}^{1}\right)^{\text {incr. }}$ by equation (39) (b can also be obtained if 
Table 1. Collections of Some Parameters and Their Volumes Adopted in This Paper

\begin{tabular}{lcc}
\hline Parameter $^{\mathrm{a}}$ & Volume & Note \\
\hline$\sigma_{f}$ and $\sigma_{m}$ & $5 * 10^{5} \mathrm{~S} / \mathrm{m}$ & conductivity \\
$\eta_{f}$ and $\eta_{m}$ & $1.6 \mathrm{~m}^{2} / \mathrm{s}$ & diffusivity \\
$\delta_{f}$ and $\delta_{m}$ & $200 \mathrm{~m}$ & thickness of the conducting layer \\
$\omega$ & diurnal & nutation frequencies \\
$B_{0}^{r}$ & 6.9 Gauss & uniform field approximation \\
\hline
\end{tabular}

${ }^{\mathrm{a}}$ Subscript $\mathrm{F}$ and $\mathrm{m}$ denote in fluid and mantle sides, respectively.

needed). Next, we use the values of all related parameters $\left(\tilde{\mathbf{X}}, \partial_{r} \tilde{\mathbf{X}}, \tilde{\mathbf{Y}}\right)$ at the fluid side of the CMB obtained in the first step without magnetic field and get a new set of $\tilde{\mathbf{X}}$ at fluid side from equation (43); and pass them to mantle side by using the new boundary condition equation (55) with equations (58)(59). We then have new values for all the variables on the mantle side. These values are different from the values that were obtained in the first step because the magnetic perturbation is now included. By using these new values of the variables on the mantle side and the variables on the fluid side obtained in the first step, one can repeat the second step until $\left(\partial_{r} R_{1}^{1}\right)^{\text {incr. }}$ ( or $\left.\mathbf{b}\right)$ converges. It turns out in practice that one iteration is enough.

[58] Once the final convergent values of all the variables on the mantle side (at the CMB) are obtained, we continue the integration from the $\mathrm{CMB}$ to the outer surface by equation (1) as usual.

[59] Once the final convergent values of all the variables on the mantle side (at the CMB) are obtained, we continue the integration from the $\mathrm{CMB}$ to the outer surface by equation (1) as usual.

[60] At a solid-fluid boundary such as CMB which is a slip boundary (if ignoring the dynamical effects, like viscosity, of the boundary layer B.L.II), the continuity condition on the displacement field only requires the normal component of $\mathbf{s}$ to be continuous, while there is not a requirement on the tangential components of $\mathbf{s}$. However, all these components are involved in the continuity conditions (equation (45) or (50)) on the stress field. Because the condition equation (45) is changed to (50), given $\tilde{\mathbf{X}}$ and $\tilde{\mathbf{Y}}$ at FOC side by standard integration in FOC (from ICB to CMB), the resulted $\tilde{\mathbf{X}}$ (including the $W_{1}^{1}$ ) and $\tilde{\mathbf{Y}}$ at mantle side will be changed significantly compared to the case without Lorentz force. It is shown in practice that the continuity conditions on the stress field at the CMB play the most important role in this coupling, while the integration of the motion equation in the thin boundary layers with the new motion equation (3) does not contribute so significantly to the nutation result.

\section{Results and Discussions}

[61] In order to compare our results with those of paper 1, we take the same values of related quantities as in paper 1 (profile B). They are: the conductivity $\sigma_{m}=\sigma_{f}=5 \times 10^{5} \mathrm{~S} / \mathrm{m}$ near the $\mathrm{CMB}$, the thickness of the conducting layer in the mantle $\delta_{m}=200 m$ and in the fluid $\delta_{f}=\delta_{m}$. Moreover, we consider only the EMC near the CMB.

[62] As to the magnetic field at $\mathrm{CMB}, B_{0}^{r}$, used in the formula of magnetic force (equation (34)) and of the $\left(\partial R_{1}^{1}\right)^{\text {incr. }}$ (equation (39)), it should be dependent on the location on the
CMB sphere, i.e., a local function of colatitude $\theta$ and longitude $\phi$. This makes the new motion equation (3) difficult to integrate. This difficulty also happens in paper 2 for the integration of $I_{b}$ in its equation (46) (or (47) for weak field approximation), although its integration is over the $\mathrm{CMB}$ surface (paper 1 does not provide the numerical values of the magnetic field). For simplicity, we use a uniform field approximation here, i.e., $B_{0}^{r}(r, \theta, \phi)=B^{\text {uniform }}$, and use the RMS value of the total field given in paper 2 as our uniform field, i.e., $B^{\text {uniform }}=6.9 G$ (Gauss). some of these used and related parameters are listed in Table 1. From the constraints of both torsional oscillations of core flow deduced from magnetic field and the length-of-day (LOD) data, regarding the variation of LOD as forced rather than free, Dumberry and Mound [2008] prefer $\tilde{B}_{c m b} \approx 3 G$. For more details see Appendix B.

[63] The results are given in Table 2. The first and second values in brackets are cited from Table 3 (profile B) of paper 1 and from Table 5 of Mathews et al. [2002] which comes from paper 2, respectively. Both values in brackets are also the contribution from EMC only happened near the CMB. The four main nutations are listed, but only 5 terms, at -18.6 year, -1 year, -0.5 year, +18.6 year and +0.5 year (plus signs and minus signs denote prograde and retrograde, respectively), are influenced by EMC. The change of the FCN period is also presented in Table 2, with the corresponding result from paper 1 in brackets, while paper 2 does not list the FCN period.

[64] As shown in Table 2, our results are of the same order as those of paper 1 when considering the same coupling constant, but differ by a factor of 1.7 for the -1 year term and a factor of 4.1 for the -18.6 year term. We will show in section 3.1 that the changes of the nutation amplitudes are consistent with the change of the FCN period in our work, and then discuss the comparison with paper 2 .

\subsection{Changes in Nutations and FCN Period}

[65] The changes in the nutation amplitudes, considering the two terms that have changed most (the -1 year and -18.6 year terms) as an example, are consistent with the change of the FCN period in this work. Note here that the FCN period obtained in this paper is subject to a small modification (about $4.5 \%$ ) of the CMB ellipticity from its hydrostatic equilibrium value [Huang et al., 2001], while in paper 1 , it is obtained for hydrostatic equilibrium CMB. This difference does not have a significant effect on the problem we discuss here.

Table 2. Effects on the Nutation Amplitudes and the FCN Period Due to EMC at the $\mathrm{CMB}^{\mathrm{a}}$

\begin{tabular}{lcccc}
\hline $\begin{array}{c}\text { Periods } \\
\text { (day) }\end{array}$ & $\begin{array}{c}\text { Pro-ip } \\
(\mu a s)\end{array}$ & $\begin{array}{c}\text { Pro-op } \\
(\mu a s)\end{array}$ & $\begin{array}{c}\text { Retro-ip } \\
(\mu a s)\end{array}$ & $\begin{array}{c}\text { Retro-op } \\
(\mu a s)\end{array}$ \\
\hline 6798.384 & $4(9,37)$ & $-4(-9,-29)$ & $-20(-83,-328)$ & $20(83,249)$ \\
365.260 & $0(0,-3)$ & $0(0,3)$ & $-39(-68,-450)$ & $39(69,411)$ \\
182.621 & $5(14,61)$ & $-5(-14,-47)$ & $-2(-3,-16)$ & $2(3,12)$ \\
13.661 & $0(0,2)$ & $0(0,-1)$ & $0(\mathrm{NA}, 0)$ & $0(\mathrm{NA}, 0)$ \\
\hline
\end{tabular}

${ }^{\mathrm{a}}$ The first and second values in parentheses are from Table 3 (profile B) of paper 1 and from Table 5 of Mathews et al. [2002], respectively. "Pro" and "retro" stand for prograde and retrograde, and "ip" and "op" stand for in-phase and out-of-phase, respectively. FCN without EMC equals -433.52 sidereal day ( -455.57$)$. FCN with EMC equals -433.14 sidereal day (-454.39). Change in the FCN period equals 0.38 sidereal day (1.18). 
Table 3. The Dependence of the Linear Coefficient $k$ in Paper 2 on the Magnetic Field Itself $\left(\left(B_{0}^{r}\right)^{2}\right)$ and on the Coriolis Force $(f)$ for Some Extreme Cases ${ }^{\mathrm{a}}$

\begin{tabular}{lcc}
\hline & $\left(B_{0}^{r}\right)^{2}=(6.9 G)^{2}$ & $\left(B_{0}^{r}\right)^{2}=(10.7 G)^{2}$ \\
\hline$f=+2 \Omega_{0}$ & $\lambda_{f+}=(4.5+5.0 i)$ & $\lambda_{f+}=(4.1+5.3 i)$ \\
$f=0$ & $k=(33.6-31.8 i)$ & $k=(34.6-30.4 i)$ \\
& $\lambda_{f+}=(3.9+5.3 i)$ & $\lambda_{f+}=(2.6+5.4 i)$ \\
$f=-2 \Omega_{0}$ & $k=(34.9-29.7 i)$ & $k=(36.2-25.2 i)$ \\
& $\lambda_{f+}=(5.3+3.9 i)$ & $\lambda_{f+}=(5.4+2.6 i)$ \\
& $k=(29.7-34.9 i)$ & $k=(25.2-36.2 i)$ \\
\hline
\end{tabular}

${ }^{\text {a }}$ The units of $k$ and $\lambda$ are $\mathrm{S} / \mathrm{m}$ and $10^{-3} \mathrm{~m}^{-1}$, respectively. If ignoring $\left(B_{0}^{r}\right)^{2}$ and $f$ in $\lambda_{f^{+}}, \lambda_{f^{+}}=(4.8+4.8 i), k=(34.6-30.4 i)$. Also, $\lambda_{s^{+}}=$ $(-4.8-4.8 i)$.

[66] Analytically, one has, as a first approximation, a direct relation between the FCN frequency change and the changes in the amplitudes of the nutations. Indeed, the prograde and retrograde nutation amplitudes can be expressed as the product of a rigid nutation amplitude and a transfer function $T(\sigma)$ accounting for the resonance effects:

$$
\begin{aligned}
T(\sigma)= & R+R^{\prime}(1+\sigma)+\frac{R_{C W}}{\sigma-\sigma_{C W}}+\frac{R_{F C N}}{\sigma-\sigma_{F C N}} \\
& +\frac{R_{F I C N}}{\sigma-\sigma_{F I C N}}+\frac{R_{I C W}}{\sigma-\sigma_{I C W}},
\end{aligned}
$$

where $\sigma$ is the frequency in the terrestrial frame expressed in cycles per sidereal day (cpsd), $R_{i}$ are the resonance strengths, and $\sigma_{i}$ the resonance frequencies in cpsd. Ignoring the inner core effect on the FCN resonance strength (neglecting a factor $A_{s} / A_{f}$ where $A_{s}$ and $A_{f}$ are the inner core and outer core moment of inertia), one can consider that $R_{F C N}$ is proportional to $\sigma_{F C N}^{\prime}=\sigma_{F C N}+1$. In that case, the effect of the changes in the amplitude of the nutation near the FCN resonance due to EMC can be approximated as

$$
\frac{\sigma_{F C N}^{\prime e m c} /\left(\sigma-\sigma_{F C N}^{e m c}\right)}{\sigma_{F C N}^{\prime n o n-e m c} /\left(\sigma-\sigma_{F C N}^{n o n-e m c}\right)} .
$$

[67] By considering that the changes in the $\mathrm{FCN}$ period induced by the EMC is $x$ days, the effects on the nutations can be considered as directly proportional to $x$ and inversely proportional to the difference between the nutation period and the FCN period in space $\left(\sim x /\left(\right.\right.$ Period $_{\text {nutation }}-$ Period $\left._{F C N}\right)$. For the annual nutation the denominator is large and the enhancement is larger too. For the 18.6 year nutation, the enhancement is almost inversely proportional to the period and therefore small. With the analytical formula, a rapid computation of the effect of the EMC at the $\mathrm{CMB}$ on the annual period shows that, for a decrease of the FCN period in space of about 0.4 day as provided by our computations, the out-of-phase components of -1 year and -18.6 year nutations increase by $41 \mu$ as and $26 \mu$ as, respectively. For a decrease of the FCN period in space of about 1.2 days (the case of the reproduction of the paper 1 results), one obtains, with the analytical formula, an increase of the -1 year nutation of $125 \mu$ as, and an increase of the -18.6 year nutation of $79 \mu$ as.

[68] From these analytical values and Table 2, we conclude that the changes of the annual and 18.6 year nutations obtained in this paper are more consistent with the change of the FCN period than in paper 1. Of course, in this estimate we ignore the effects on the other parameters of the transfer function. However, these are expected to be small if EMC is considered only at the CMB.

\subsection{On the Contribution of the Energy of the Magnetic Field and the Coriolis Force to the Coefficient $k$}

[69] In this section, we want to make some, but not full, discussion on how many factors play roles in the change of the final nutations and how much they influence. These factors include the energy of the magnetic field and the contribution of the Coriolis force.

[70] Comparing the results of paper 1 with those of paper 2 (listed in Table 5 of Mathews et al. [2002]), we see that the changes of the main nutations in paper 1 are smaller than those of paper 2 by a factor of about 6 for the -1 year nutation and approximately 4 for the -18.6 year nutation. paper 1 assumes that the energy other than the dipole magnetic field increase 5 times, and paper 2 seeks the Lorentz force and contribution from the magnetic field near the ICB.

[71] The results in paper 2 [also Mathews et al., 2002] are improved in several ways comparing to paper 1 . The effects of the Coriolis force was included, and the study by Mathews et al. [2002] also included the back reaction of the Lorentz force on the fluid flow. Adding the Lorentz force alters the velocity of the fluid core at the core-mantle boundary. And the energy of the magnetic field is also an important parameter, as expected in paper 1 that increasing the magnetic energy by 4 times, via the part of short wavelength, may increase the final nutation terms by similar times.

[72] From equation (34), $L$ (and the magnetic torque) obviously depends on both $k$ and $\left(B_{0}^{r}\right)^{2}$, and it is clear that the effects of changing the magnetic field have two parts: (1) direct, in changing the value of $\left(B_{0}^{r}\right)^{2}$, and (2) indirect, in changing the value of coefficient $k$ and changing the value of $\mathbf{s}_{f-m}$ compared to displacement $\mathbf{s}$. The "back reaction" affects only the second kind of these two changes.

[73] It is consistent, rather than conflict, with the formulae of the torque used in both paper 1 and paper 2 that are cited from early papers (Rochester, 1962, 1976). $k$ is only a linear coefficient in equation (34) and it is independent on $B_{0}^{r}$ in paper 1 , but it depends on both Coriolis force and $B_{0}^{r}$ in paper 2 .

[74] Because the Lorentz force is almost linearly proportional to the coefficient $k$ additional to $\left(B_{0}^{r}\right)^{2}$, we will calculate this $k$ in this section and show that, if not considering the change of the boundary conditions as section 2.4 , neither the including Coriolis force nor increasing the magnetic field contributes much to $k$ (not the final nutation here). However, one should keep in mind that the following analysis is only an approximate comparison.

[75] On the one hand, for equation (27) related to paper 1, one can substitute the quantities with the values given in paper 1, i.e., $\eta_{m}=\eta_{f}=1.6 \mathrm{~m}^{2} / \mathrm{s}, \delta_{m}=200 \mathrm{~m}$ (for $\omega=\Omega_{0}$ ), and $\delta_{f}=\delta_{m}$, and then get $k=(32.7-32.7 i)$.

[76] On the other hand, for equation (30) related to paper 2, we discuss some extreme cases to be compared with the result in paper 1 .

[77] First, if we ignore the Coriolis force, then $f=$ $2 \Omega_{0} \cos \theta \rightarrow 0$. Moreover, if $\frac{\left(B_{0}^{r}\right)^{2}}{\mu_{0} \rho} \ll \eta_{f} \omega$, i.e., $\frac{\sqrt{2} B_{0}^{r}}{\sqrt{\mu_{0} \rho} \omega \delta_{f}} \ll 1$, 
then $\lambda_{f^{+}}^{2} \rightarrow i \omega / \eta_{f}$ and $\lambda_{f^{+}} \rightarrow+\sqrt{i \omega / \eta_{f}}$ for the CMB. After substituting this into equation (30) and noticing that $\lambda_{s}=$ $-\sqrt{i \omega / \eta_{s}}$ for the CMB. One finds that equation (30) derived in paper 2 exactly degenerates to equation (27) which is obtained in paper 1. Or from another comparison of equation (27) for paper 1 with equation (30) for paper 2, we see that $\lambda_{s}$ in equation (30) acts as $\delta_{s}$ in equation (27), and that $\lambda_{f}$ in equation (30) acts as $\delta_{f}$ in equation (27). Moreover, in equation (30), the value of $\lambda_{f}$ is of the same order of magnitude as $\lambda_{s}$. Therefore, $k_{B 1992}=$ $k_{B 2002}$ if $\delta_{f}=\delta_{s}$.

[78] Second, we compute equation (30) by keeping the contribution from $\left(B_{0}^{r}\right)^{2}$ and the Coriolis force (i.e., $\left.f\right)$ in $\lambda_{f+}$, and consider three extreme cases for $f=2 \Omega_{0} \cos \theta: f=2 \Omega_{0}$, $f=0$, and $f=-2 \Omega_{0}$. Moreover, in order to see the contribution of $\left(B_{0}^{r}\right)^{2}$ clearly, we consider two cases: $\left(B_{0}^{r}\right)^{2}=$ $(6.9 G)^{2}$ (paper 2 takes the RMS of the total magnetic field at $\mathrm{CMB}$ as $6.9 G$ ) and $\left(B_{0}^{r}\right)^{2}=(10.7 G)^{2}$ (the magnetic energy of the latter one is approximately double of the former one). Table 3 lists the results for $k$ and $\lambda_{f+}$, in which $\omega \approx \Omega_{0}$ for diurnal nutations, $\eta_{m}=\eta_{f}=1.6 \mathrm{~m}^{2} / \mathrm{s}$ and $\rho=\rho_{f}=$ $9.9 * 10^{3} \mathrm{~kg} / \mathrm{m}^{3}$.

[79] From Table 3, one can conclude (1) that increasing magnetic energy by doubling can increase $\lambda_{f+}$ and $k$ by about $20 \%$ only, (2) that the contributions of the Coriolis force to $k$ is also smaller than about $20 \%$, and (3) that the total back reaction contributions of both the Coriolis force and magnetic field to the linear coefficient $k$ ( $k$ only, rather than $\mathbf{L}$ or torque and thus the final nutation) is smaller than $40 \%$.

[80] It means that, if ignoring the different treatment of magnetic field (dipole filed only, uniform field only, or total energy) in paper 1 and in paper 2 and taking the same magnetic energy, the direct contributions of both the Coriolis force and magnetic field itself to the linear coefficient $k$ is smaller than $40 \%$.

[81] From this calculation, it is shown that the big improvement of the -1 year nutation in paper 2 from paper 1 , i.e., get a much larger number $(0.4$ mas $)$ which is required to fill the gap between the VLBI observation and classical theoretical calculation is not mainly due to the inclusion of back reaction contributions of both the Coriolis force and magnetic field itself, as implied in paper 2 . There must be other reasons.

[82] On the one hand, paper 1 does not give the value of $B_{0}^{r}$, while paper 2 gives the overall root-mean-square (RMS) of the radial field at CMB as $6.9 \mathrm{G}$ (partitioned into $2.64 \mathrm{G}$ for dipole and $6.4 \mathrm{G}$ for other nondipole component), we cannot guess how much paper 1 adopts but they should be similar although paper 1 states that the magnetic energy requires to enlarge 4 times.

[83] However, from the analysis in Appendix B, it is shown that paper 1 used only the terms of $l=n$ and $m=k$ in magnetic field and excluded other cross terms in evaluating the magnetic torque $\boldsymbol{\Gamma}$. However, these cross terms do not cancel and should be included in the calculation of $\boldsymbol{\Gamma}$. The resulting $\Gamma$ will not have the simple relationship with $\Omega_{f}$ as in equation (53) of paper 1 and will become a very complex calculation. Furthermore, Appendix $\mathrm{C}$ shows that, if using the same magnetic field and the weak field approximation, paper 1 and paper 2 should get the same magnetic torque on the mantle, and therefore the same magnetic coupling constant $K^{c m b}$ (and thus nutation) at CMB.

[84] On the other hand, we use also $6.9 \mathrm{G}$ as in paper 2, the results in Table 2 is smaller than that of paper 2 by approximately 1 order of magnitude.

[85] Finally, if a double magnetic energy $\left(\left(B_{0}^{r}\right)^{2}\right)$ is applied in all the Lorentz force in equation (34), boundary conditions (equation (55)) and $k$ itself, rather than applied to $k$ only as done in this section, the final entire -1 year nutation will be increased by approximately 1.8 .

\subsection{Brief Remarks}

[86] In this paper, we have calculated the effects of EMC at the $\mathrm{CMB}$ on nutations by numerical integration. Both the Lorentz force and the Coriolis force are integrated in this integration; the back reaction (a real "coupling" between magnetic field and nutation) is also included automatically in this dynamic system by two points: (1) the velocity field of the fluid core near the CMB is changed, via the change of the ODE, which is described in detail in the content of the section 2.3, and (2) the boundary conditions cross the CMB are also changed, which is described in detail in the text of the section 2.4. Following the above two steps, the velocity field of the particles in the mantle side near CMB, and thus the final nutation terms, is therefore also changed.

[87] The formula of the Lorentz force and the new boundary conditions are derived, a strategy (iteration) to solve this coupling system is given in detail in section 2.5.

[88] Using the solution of the induced magnetic field $\mathbf{b}$ and the same values of the related parameters as in paper 1, and the energy of the reference magnetic field being taking the same as paper 2 (as it is not given directly in paper 1), we get results listed in Table 2: the FCN period decreases by 0.38 day, and the out-of-phase (in-phase) amplitudes of the -18.6 year and the -1 year nutations increase (decrease) by 20 and $39 \mu a s$, respectively. They are of the same order as those of paper 1, but approximately 1 order of magnitude smaller than required to fill the gap in the out-of-phase component of the -1 year nutation between the VLBI observation and the theoretical value.

[89] A discussion on the contribution of the energy of the magnetic field and the Coriolis force to the coefficient $k$ (and thus to $K^{c m b}$ ) is also presented in section 3.2. A comparison with the results of paper 1 and paper 2 is made and differences remain, and it shows that more in-depth studies are needed for this problem, especially the out-ofphase component of the -1 year nutation.

[90] For example, Jackson and Livermore [2009] point out that the formal lower bound for the Ohmic dissipation based on all possible (including geodetic) constraints and on a 3-D magnetic field configuration is almost $10 \mathrm{GW}$, that is well below estimates, from geodynamo models, of the energy needed to power the dynamo, which are around several TW.

[91] The results concerning the magnetic coupling effects on nutation (in particular, the RMS magnetic field at the core boundaries) presented in paper 2 may be considered as upper bounds, as other coupling mechanisms may be significant at the core boundary. Viscous torque (considering an effective viscosity) is another potential coupling mechanism and can be used to explain the difference between the observed nutations and the theoretical model [Mathews and Guo, 2005; Deleplace and Cardin, 2006], although Buffett 
and Christensen [2007] suggest that viscous coupling can only explain a few percent of the dissipative torque between the core and the mantle. More discussions on the viscosity and torsional oscillations can also be found in the work by, e.g., Mound and Buffett [2005, 2007] and Palmer and Smylie [2005]. Topographic torque maybe important as well as proposed by Wahr and de Vries [1989], Wu and Wahr [1997] and Folgueira and Dehant [2008].

[92] Regarding to the reference configuration with the magnetic field $\mathbf{B}_{0}$ as described at the second paragraph after equation (2), another possible explanation on the dynamical consistencies of $\mathbf{B}_{0}$ and $\mathbf{J}_{0}$ may be, from numerical dynamo simulations, that $\mathbf{B}_{0}$ is to leading order a potential field in the mantle, and in this case, $\mathbf{J}_{0} \approx 0$. If so, one does not need to discuss the likely mechanisms how to offset a finite background magnetic torque from $\mathbf{B}_{0}$ and $\mathbf{J}_{0}$ (from the comments of anonymous reviewer). For detail discussion of likely mechanisms to offset a finite background magnetic torque from the magnetic field and current, readers are recommended to refer related MHD studies. Anyhow, it would be worth trying to look at nutation as a small perturbation on a numerical geodynamo model in the future study. The numerical geodynamo models are all done in spherical geometry, and one problem to be solved is to decide what ellipsoidal shape of the CMB would be reasonably consistent with such a model rather than with the usual hydrostatic equilibrium model.

[93] Regarding to the boundary condition, The formulation in this paper follows that of Smith [1974]. The boundary conditions on elliptical surfaces are expressed on an equivalent spherical surface using a Taylor series. When the boundary displacement due to ellipticity is small then it is reasonable to truncate the Taylor series after the first-order terms. However, when this procedure is applied to the a small perturbation near $\mathrm{CMB}$, like the magnetic perturbation here, one should be careful because the magnetic boundary layer is only a few hundred meters thick. Especially for the FCN and -1 year nutation, they are very sensitive to the physics near CMB, i.e., the boundary situation. Our practical calculation show that any small change in the boundary condition at $\mathrm{CMB}$ will cause a large (at least not ignorable) change in the displacement field (and thus the final nutation) comparing with the "direct" effect of the Lorentz force. Another example is that, when discussing possible couplings between rotational modes and core modes, Rogister [2003] and Rogister and Valette [2009] point out that the coupling chain in the expression of the displacement field in the FOC should be truncated at higher degree, i.e., include one (even two) more term. The further study of how this factor influence the coupling between nutation and magnetic field near $\mathrm{CMB}$ will be also interesting.

\section{Appendix A: Dimensional Analysis and Approximations}

[94] In order to simplify the computation, in what follows we present some justifications for the approximations and assumptions used in the text.

[95] 1. The total velocity field of fluid is $\mathbf{v}_{0}+\mathbf{v}$, where $\mathbf{v}_{0}$ is the reference velocity field in the core supporting $\mathbf{B}_{0}$ for its long-term component, and $\mathbf{v}$ is mainly the relative nutational motion of the FOC to the mantle or to the SIC and describes the entire nutation relative to the adopted steadily rotating reference frame. They may be of the same order (e.g., $\left|\mathbf{v}_{0}\right| \sim 0.5 \mathrm{~mm} / \mathrm{s} ;|\mathbf{v}| \sim 0.1 \mathrm{~mm} / \mathrm{s}$ ).

[96] 2. As shown in the text, there is no radial component in both $\mathbf{b}$ and $\mathbf{v}$.

[97] 3. The length scales of $\mathbf{B}_{0}$ and $\mathbf{v}_{0}\left(L_{B 0}\right.$ and $\left.L_{V 0}\right)$ are assumed to be comparable to the radius of the core, $a_{c}$ (or $a_{i c b}$ near ICB).

[98] 4. Here $\mathbf{b}$ is confined to the viscous layer at the fluid side and to the conducting layer at the solid side. Their thickness or boundary thickness is much smaller than the radius of the boundary; the length scale of $\mathbf{b}, \delta_{b}$, depends on the magnetic skin depth and is likely shorter than $1 \mathrm{~km}$.

[99] 5. Taking the FOC as steady rotating reference frame, it is assumed, on the fluid side, that $\mathbf{v}$ is confined to the thin boundary layers and that $\mathbf{v}$ varies in the radial direction from $\mathbf{v}=\omega_{f} \times \mathbf{r}$ at the interface to zero, over distances of the viscous boundary layer, which is very small (even possibly a few centimeters). The length scale of $\mathbf{v}, \delta_{v}$, in the vicinity of the fluid core boundaries depends on the boundary layers and has an upper bound of $\delta_{b}$.

[100] 6. Because of the above two arguments, the horizontal derivatives of $\mathbf{b}$ and $\mathbf{v}$ are much smaller than their radial derivatives, i.e., $\partial_{r} \mathbf{v} \gg\left|\nabla_{H} \mathbf{v}\right|, \partial_{r} \mathbf{b} \gg\left|\nabla_{H} \mathbf{b}\right|$, where $\nabla_{H} \equiv \frac{1}{r} \nabla_{1} \equiv \frac{1}{r}\left[\hat{\theta} \partial_{\theta}+\hat{\varphi} \frac{1}{\sin \theta} \partial_{\varphi}\right]$.

[101] 7 . Because $\mathbf{b}$ and $\mathbf{v}$ vary rapidly with radius in the thin boundary layers, whose thickness is much smaller than the radius of core, we conclude $\frac{1}{r} \partial_{r} \mathbf{b} \ll \partial_{r}^{2} \mathbf{b}, \frac{1}{r} \partial_{r} \mathbf{v} \ll \partial_{r}^{2} \mathbf{v}$.

[102] 8. Now we may compare the terms in the following expansions

$$
\begin{aligned}
& \nabla \times\left(\mathbf{v}_{0} \times \mathbf{b}\right)=\mathbf{b} \cdot \nabla \mathbf{v}_{0}-\mathbf{v}_{0} \cdot \nabla \mathbf{b}+\mathbf{v}_{0}(\nabla \cdot \mathbf{b})-\mathbf{b}\left(\nabla \cdot \mathbf{v}_{0}\right) \\
& \nabla \times\left(\mathbf{v} \times \mathbf{B}_{0}\right)=\mathbf{B}_{0} \cdot \nabla \mathbf{v}-\mathbf{v} \cdot \nabla \mathbf{B}_{0}+\mathbf{v}\left(\nabla \cdot \mathbf{B}_{0}\right)-\mathbf{B}_{0}(\nabla \cdot \mathbf{v}) .
\end{aligned}
$$

From the solenoidal conditions of $\mathbf{b}$ and $\mathbf{B}$, the two related terms in both equations can be removed. It is conventionally assumed, when making geomagnetic dynamo models, that $\nabla \cdot \mathbf{v}_{0}=0$. This is valid for long-period changes in the flow such as those associated with a dynamo. While for shorter periods, say 1 day or shorter when measured in the rotating frame, it is necessary to take a finite (but large) Lame parameter $\lambda$ into account, and to allow $\nabla \cdot \mathbf{v}$ to be nonzero. This is what is done in the usual treatments of wobble/ nutation. However, noting the assumption that $\mathbf{v}$ (and $\mathbf{b}$ as well) has no radial component, and that the horizontal derivatives of $\mathbf{v}$ are small compared with the radial derivative, one can still easily ignore $\mathbf{B}_{0}(\nabla \cdot \mathbf{v})$ compared with $\mathbf{B}_{0} \cdot \nabla \mathbf{v}$. Moreover, the length scales of the remaining four terms are: $\mathbf{B}_{0} \cdot \nabla \mathbf{v} \sim B_{0} v / \delta_{v} ; \mathbf{v} \cdot \nabla \mathbf{B}_{0} \sim B_{0} v / L_{B 0} ; \mathbf{v}_{0} \cdot \nabla \mathbf{b} \sim b v_{0} / \delta_{b} ; \mathbf{b} \cdot$ $\nabla \mathbf{v}_{0} \sim b v_{0} / L_{V 0}$. Therefore, if one considers $\mathbf{B}_{0} \cdot \nabla \mathbf{v}$ as being of the first order, then the second and third terms are regarded as second order, and the last term as third order, which has been discussed by Buffett [1993]. This explains why the sum of equations (A1) and (A2) can be written as

$$
\nabla \times\left(\mathbf{v}_{0} \times \mathbf{b}\right)+\nabla \times\left(\mathbf{v} \times \mathbf{B}_{0}\right) \approx \mathbf{B}_{0} \cdot \nabla \mathbf{v}
$$


[103] 9. In the thin boundary layers, $\partial_{r} B_{0}^{r} \approx-\frac{l+2}{r} B_{0}^{r} \approx-\frac{l+2}{a_{c}} B_{0}^{r}$ for the degree $l$ component of the magnetic field, while $\partial_{r} \mathbf{v}_{f-m} \approx \frac{\mathbf{v}_{f-m}}{\delta_{m}}$, and $\frac{l+2}{a_{c}} \ll \frac{1}{\delta_{m}}$ if the degree $l$ is not too large. Therefore, $\partial_{r} B_{0}^{r} \mathbf{v}_{f-m}$ can be ignored compared with $B_{0}^{r} \partial_{r} \mathbf{v}_{f-m}$.

\section{Appendix B: On the Magnetic Torque $\Gamma_{m}^{(b)}$ in paper 1}

[104] The magnetic torque $\Gamma_{m}^{(b)}$ on the mantle at the CMB is (see equation (48) in paper 1 or equation (15) of Rochester [1962]):

$$
\Gamma_{m}^{(b)}=\frac{1}{\mu_{0}} \int_{S}(\mathbf{r} \times \mathbf{b}) B_{0}^{r} d S .
$$

If the CMB is assumed spherical then $\hat{\mathbf{r}}=\hat{\mathbf{n}}$ on it (in the notation system in the work of Rochester [1962], $\hat{\mathbf{r}}=-\hat{\mathbf{n}})$.

[105] Because $\mathbf{b}=k B_{0}^{r} \mathbf{v}_{f-m}$ where $k$ is $-k_{1}$ or $-k_{2}$ or $-k_{3}$ in section 2.2, and considering that

$$
\mathbf{v}_{f-m}=\left(\boldsymbol{\Omega}_{f}-\boldsymbol{\Omega}_{m}\right) \times \mathbf{r}=\boldsymbol{\Omega}_{f} \times \mathbf{r}
$$

(if $\boldsymbol{\Omega}_{m}=0$ in the coordinate system fixed with the rotating mantle), and that $\boldsymbol{\Omega}_{f}$ for nutation has only equatorial components, then

$$
\begin{aligned}
\boldsymbol{\Gamma}_{m}^{(b)} & =\frac{k}{\mu_{0}} \oint_{S}\left[B_{0}^{r}\right]^{2}\left[\mathbf{r} \times\left(\boldsymbol{\Omega}_{f} \times \mathbf{r}\right)\right] d S \\
& =\frac{k}{\mu_{0}}\left\{\oint_{S}\left[B_{0}^{r}\right]^{2} r^{2} \boldsymbol{\Omega}_{f} d S-\oint_{S}\left[B_{0}^{r}\right]^{2}\left(\mathbf{r} \cdot \boldsymbol{\Omega}_{f}\right) \mathbf{r} d S\right\} \\
& =\frac{k}{\mu_{0}}\left(A_{1}-A_{2}\right),
\end{aligned}
$$

which is equation (52) of paper 1.

\section{B1. The First Part of the Integral, $A_{1}$}

[106] Since $\boldsymbol{\Omega}_{f}$ has only equatorial components,

$$
\Omega_{f}=\Omega_{1} \hat{x}+\Omega_{2} \hat{y}
$$

then

$$
A_{1}=\oint_{S}\left[B_{0}^{r}\right]^{2}\left(r^{2} \boldsymbol{\Omega}_{f}\right) d S=a_{c}^{2} \boldsymbol{\Omega}_{f} \oint_{S}\left[B_{0}^{r}\right]^{2} d S
$$

[107] From the definition of $B_{0}^{r}$ at $r=a_{c}$

$$
B_{0}^{r}\left(a_{c}\right)=\sum_{l=1}^{N} \sum_{m=0}^{l}\left[C_{l}^{m}\left(a_{c}\right) \cos m \phi+S_{l}^{m}\left(a_{c}\right) \sin m \phi\right] P_{l}^{m}(\cos \theta),
$$

where the definition of the associated Legendre function $P_{l}^{m}$ in paper $1 \mathrm{can}$ be retrieved from its normalization formula that is his formula (50) (corrected). Defining

$$
P_{l}^{m}(x)=\left(\frac{1}{2^{n} n !}\right)\left(1-x^{2}\right)^{m / 2}\left(\frac{d}{d x}\right)^{l+m}\left(x^{2}-1\right)^{l}
$$

then the normalization factor of $P_{l}^{m}$ should be

$$
\oint_{S 1} P_{l}^{m}(\cos \theta) P_{n}^{m}(\cos \theta) d S=\frac{4 \pi \delta_{l n}}{2 l+1} \frac{(l+m) !}{(l-m) !},
$$

$$
\begin{aligned}
M_{l}^{m} & =\oint_{S 1} P_{l}^{m}(\cos \theta) \cos (m \phi) P_{n}^{k}(\cos \theta) \cos (k \phi) d S \\
& =2 \pi \delta_{l n} \delta_{m k} \frac{\left(1+\delta_{0 m}\right)(l+m) !}{(2 l+1)(l-m) !}
\end{aligned}
$$

$$
\begin{aligned}
& \oint_{S 1} P_{l}^{m}(\cos \theta) \sin (m \phi) P_{n}^{k}(\cos \theta) \sin (k \phi) d S \\
& =2 \pi \delta_{l n} \delta_{m k} \frac{\left(1-\delta_{0 m}\right)(l+m) !}{(2 l+1)(l-m) !}, \\
& \oint_{S 1} P_{l}^{m}(\cos \theta) \cos (m \phi) P_{n}^{k}(\cos \theta) \sin (k \phi) d S=0,
\end{aligned}
$$

where the integral surface $S 1$ is a unit sphere surface of radius $r=1$.

[108] There is a mistake in equation (50) of paper 1 compared with our equation (B10). Fortunately, noting that all $S_{l}^{0} \equiv 0$, this mistake does not affect the calculation of $\oint\left[B_{0}^{r}\right]^{2} d S$ and $\boldsymbol{\Gamma}$. Then

$$
\oint_{S}\left[B_{0}^{r}\right]^{2} d S=\left(a_{c}\right)^{2} \sum_{l} \sum_{m} M_{l}^{m}\left[B_{l}^{m}\right]^{2},
$$

where

$$
\left[B_{l}^{m}\right]^{2}=\left[C_{l}^{m}\right]^{2}+\left[S_{l}^{m}\right]^{2}
$$

[109] Therefore, the first part of $\boldsymbol{\Gamma}_{m}^{(b)}$ in equation (B3) is

$$
\frac{k}{\mu_{0}} a_{c}^{4} \boldsymbol{\Omega}_{\mathbf{f}} \sum_{l, m} M_{l}^{m}\left[\left(C_{l}^{m}\right)^{2}+\left(S_{l}^{m}\right)^{2}\right]
$$

(Note: it is $M_{l}^{m}$ in the above equation rather than $N_{l}^{m}$ as in equation (53) of paper 1).

\section{B2. Numerical Value of $\oint_{\boldsymbol{s}}\left[B_{0}^{r}\right]^{2} d S$}

[110] $\oint_{S}\left[B_{0}^{r}\right]^{2} d S$ represents the total flux of the magnetic energy over the spherical surface of the boundary, which can also be seen in the following definition. Although no final numerical result of $\oint_{S}\left[B_{0}^{r}\right]^{2} d S$ is given in paper 1 , one can still find the detailed description of how to get it from a given geomagnetic field model which is usually given at the Earth surface and represented by spherical harmonic expansion.

[111] From the IGRF2000 model [International Association of Geomagnetism and Aeronomy Division V, Working Group $8,2000]$, the geomagnetic potential is given at the Earth's surface $(r=a)$ by

$$
V(\mathbf{r})=\sum_{l=1}^{N} \sum_{m=0}^{l}\left[g_{l}^{m}(r) \cos m \phi+h_{l}^{m}(r) \sin m \phi\right] P_{l}^{m}(\cos \theta),
$$


where $P_{l}^{m}$, different from that given in above, is the Schmidt quasi-normalized associated Legendre function defined by

$$
P_{l}^{m}(x)=\sqrt{\frac{2(l-m) !}{\left(1+\delta_{0 m}\right)(l+m) !}}\left(\frac{1}{2^{m} m}\right) \times\left(1-x^{2}\right)^{m / 2}\left(\frac{d}{d x}\right)^{l+m}\left(x^{2}-1\right)^{l} .
$$

[112] From $\mathbf{B}=-\nabla V$ and downward continuation of the radial field from the Earth's surface $(r=a)$ to the boundary $\left(r=a_{c}\right)$, ignoring the work of Ballani et al. [2002] in which they indicate that the spherical harmonical downward extrapolate in the mantle may be not suitable for $\mathbf{B}$ to get $B_{C M B}$, we get the radial magnetic field at the boundary

$$
B_{0}^{r}=\sum_{l=1}^{N} \sum_{m=0}^{l}\left[C_{l}^{m} \cos m \phi+S_{l}^{m} \sin m \phi\right] P_{l}^{m}(\cos \theta),
$$

where

$$
\left\{\begin{array}{c}
C_{l}^{m} \\
S_{l}^{m}
\end{array}\right\}=(l+1)\left(\frac{a}{a_{c}}\right)^{l+2} *\left\{\begin{array}{l}
g_{l}^{m} \\
h_{l}^{m}
\end{array}\right\} .
$$

By the definition, at $r=a_{c}$,

$$
\begin{aligned}
\overline{\left(B_{0}^{r}\right)^{2}} \equiv & \oint_{c m b}\left[\sum_{l=1}^{N} \sum_{m=0}^{l}\left(C_{l}^{m} \cos m \phi+S_{l}^{m} \sin m \phi\right) P_{l}^{m}(\cos \theta)\right] \\
& *\left[\sum_{l^{\prime}=1}^{N} \sum_{m^{\prime}=0}^{l^{\prime}}\left(C_{l^{\prime}}^{m^{\prime}} \cos m^{\prime} \phi+S_{l^{\prime}}^{m^{\prime}} \sin m^{\prime} \phi\right) P_{l^{\prime}}^{m^{\prime}}(\cos \theta)\right] d S .
\end{aligned}
$$

By using the orthogonality of $P_{l}^{m}$, and noticing that $S_{l}^{0} \equiv 0$, we get

$$
\overline{\left(B_{0}^{r}\right)^{2}}=\sum_{l=1}^{N} \sum_{m=0}^{l}\left[\left(C_{l}^{m}\right)^{2}+\left(S_{l}^{m}\right)^{2}\right] * \frac{4 \pi}{2 l+1} .
$$

[113] Using the same above procedure as given in paper 1 and the IGRF2000 model [International Association of Geomagnetism and Aeronomy Division V, Working Group 8, 2000] for the J2000.0 epoch, in which the truncated degree/ order is $10 / 10$, and having noticed that the definition and normalization factor of the associated Legendre functions used in the spherical harmonics expansion of the radial magnetic field in paper 1 is different from the Schmidt quasinormalized associated Legendre function used in IGRF2000 model, we get $\left(B_{0}^{r}\right)^{2} \approx(10.7 G)^{2}$ at the CMB.

\section{B3. The Second Part of the Integral, $A_{2}$}

[114] Evaluation of $A_{2}$ in equation (B3) is a little cumbersome:

$$
\begin{aligned}
A_{2} & \equiv \oint_{S}\left[B_{0}^{r}\right]^{2}\left(\mathbf{r} \cdot \boldsymbol{\Omega}_{f}\right) \mathbf{r} d S \\
& =\oint_{S}\left[B_{0}^{r}\right]^{2} r^{2}\left\{\begin{array}{l}
\sin ^{2} \theta\left(\Omega_{1} \cos ^{2} \phi+\Omega_{2} \sin \phi \cos \phi\right) \hat{x} \\
+\sin ^{2} \theta\left(\Omega_{1} \sin \phi \cos \phi+\Omega_{2} \sin ^{2} \phi\right) \hat{y} \\
+\sin \theta \cos \theta\left(\Omega_{1} \cos \phi+\Omega_{2} \sin \phi\right) \hat{z}
\end{array}\right\} d S .
\end{aligned}
$$

Its x component,

$$
\left(A_{2}\right)_{x}=a_{c}^{4} \sum_{l, m} \sum_{n, k} G_{l n}^{m k} \times H_{l n}^{m k},
$$

where

$$
\begin{aligned}
H_{l n}^{m k} \equiv & \int_{0}^{2 \pi}\left(\Omega_{1} \cos ^{2} \phi+\Omega_{2} \sin \phi \cos \phi\right) \\
& \times\left(C_{l}^{m} \cos m \phi+S_{l}^{m} \sin m \phi\right)\left(C_{n}^{k} \cos k \phi+S_{n}^{k} \sin k \phi\right) d \phi \\
= & \begin{cases}\pi \Omega_{1}\left(C_{l}^{m} C_{n}^{k}\right) & \text { if } k=m=0 \\
\frac{\pi}{2} \Omega_{1}\left(C_{l}^{m} C_{n}^{k}+S_{l}^{m} S_{n}^{k}\right) & \text { if } k=m \neq 0, \neq 1 \\
\frac{\pi}{4}\left[\begin{array}{ll}
\Omega_{1}\left(C_{l}^{m} C_{n}^{k}-S_{l}^{m} S_{n}^{k}\right) \\
+\Omega_{2}\left(C_{l}^{m} S_{n}^{k}+S_{l}^{m} C_{n}^{k}\right)
\end{array}\right] & \text { if } m+k=2 \\
\frac{\pi}{4}\left[\begin{array}{ll}
\Omega_{1}\left(C_{l}^{m} C_{n}^{k}+S_{l}^{m} S_{n}^{k}\right) \\
\pm \Omega_{2}\left(S_{l}^{m} C_{n}^{k}-C_{l}^{m} S_{n}^{k}\right)
\end{array}\right] & \text { if } m-k= \pm 2 \\
0 & \text { else }\end{cases}
\end{aligned}
$$

and

$$
G_{l n}^{m k} \equiv \int_{0}^{\pi} P_{l}^{m} P_{n}^{k} \sin ^{3} \theta d \theta
$$

[115] The analytic result of $G_{l n}^{m k}$ is unavailable in literature and not easy to get, however, we can get it if $l=n$ and $m=k$ (see Appendix (E)):

$$
\begin{aligned}
G_{l l}^{m m}= & \left\{1-\frac{(l+m)(l-m)}{(2 l+1)(2 l-1)}-\frac{(l+m+1)(l-m+1)}{(2 l+1)(2 l+3)}\right\} \\
& * \frac{2}{2 l+1} \frac{(l+m) !}{(l-m) !} .
\end{aligned}
$$

[116] Even though we cannot get the analytic result of $G_{l n}^{m k}$ for all $l, n, m, k$, we can still calculate them numerically.

\section{B4. Summary and Discussion of $\Gamma_{m}^{(b)}$ in Paper 1}

[117] From the numerical values of $G_{l n}^{m k}=\int_{0}^{\pi} P_{l}^{m} P_{n}^{k} \sin ^{3} \theta d \theta$, one can see that $G_{l n}^{m k} \neq 0$ for many $m+k=2$ and $m-k= \pm 2$, which means that both $\Omega_{1}$ and $\Omega_{2}$ are included in the $x$ (and $y$ ) component of $A_{2}$ in equation (B21), so $A_{2}$ (and therefore $\Gamma_{m}^{(b)}$ ) do not have a simple relation with $\Omega_{f}$ as in paper 1 , in which $\Gamma_{m}^{(b)}$ was parallel to $\Omega_{f}$.

[118] However, if we keep only the terms $l=n$ and $m=k$, then

$$
\Gamma_{m}^{(b)}=\frac{k}{\mu_{0}}\left(A_{1}-A_{2}\right)=\frac{k}{\mu_{0}} a_{c}^{4} \boldsymbol{\Omega}_{\mathbf{f}} \sum_{l, m} d_{l}^{m}\left[\left(C_{l}^{m}\right)^{2}+\left(S_{l}^{m}\right)^{2}\right],
$$

where

$$
\begin{aligned}
d_{l}^{m} & =M_{l}^{m}-\frac{\pi}{2}\left(1+\delta_{0 m}\right) G_{l l}^{m m} \\
& =\frac{M_{l}^{m}}{2}\left[1+\frac{(l+m)(l-m)}{(2 l+1)(2 l-1)}+\frac{(l+m+1)(l-m+1)}{(2 l+1)(2 l+3)}\right],
\end{aligned}
$$

which is exactly the $N_{l}^{m}$ defined by equation (55) in paper 1 . 


\section{B5. Conclusion}

[119] Based on the above analysis, we can conclude that paper 1 used only the terms $l=n$ and $m=k$ and excluded other cross terms in evaluating the torque $\boldsymbol{\Gamma}$. However, these cross terms do not cancel and should be included in the calculation of $\boldsymbol{\Gamma}$. The resulting $\boldsymbol{\Gamma}$ will not have the simple relationship with $\Omega_{f}$ as in equation (53) of paper 1 and will become a very complex calculation.

\section{Appendix C: Remarks on $B_{0}^{r}$ and Proof of the Equivalence Between $\Gamma_{P 1}$ and $\Gamma_{P 2}$}

[120] As pointed out in the text, the radial magnetic field at CMB $\left(B_{0}^{r}\right)$, used in the formula for the Lorentz force (equation (34)) and for the $\left(\partial_{r} R_{1}^{1}\right)^{\text {incr. }}$ (equation (39)), should depend on the location on the CMB sphere (i.e., a local function of colatitude $\theta$ and longitude $\phi$ ). This makes the new motion equation (3) difficult to integrate, and a full (or approximated) model of $B_{0}^{r}$ is then needed. This difficulty also arises in paper 2 for the integration of $I_{b}$ in equation (46) (or (47) for weak field approximation). In paper 2, the magnetic field $B_{0}^{r}$ is separated into two configurations for simplicity.

[121] 1. The dipole field represents the dominant longwavelength component, this part has a simple dependence of $\theta: B_{r}^{\text {dipole }}(r, \theta, \phi)=B_{p} \cos \theta$, where $B_{p}$ is a constant (independent on $\theta$ and $\phi$ ).

[122] 2. The other multipole components represent the shorter-wavelength field except the dipole field, and this part makes the integration difficult.

[123] In order to deal with this, paper 2 introduces a concept "uniform field," $B_{r}^{\text {uniform }}$. This uniform field is independent of $\theta$ and $\phi$, i.e., a constant everywhere on the CMB, so that it can be moved out from the integral as done for $B_{p}$. Moreover, the strength of this uniform field is determined by the requirement that $\left(\overline{B_{r}^{\text {dipole }}}\right)^{2}+\left(\overline{B_{r}^{\text {uniform }}}\right)^{2}=\left(\overline{B_{r}^{\text {total }}}\right)^{2}=$ $(6.9 G)^{2}$, where, $\overline{B_{r}^{\text {dipole }}}, \overline{B_{r}^{\text {uniform }}}$ and $\overline{B_{r}^{\text {total }}}$ are the so-called "root-mean-square" (RMS) values of the dipole field, RMS of the uniform field, and RMS of the total field, respectively. paper 2 declares that, in order to get the required torque (and then the coupling constant) for the wanted nutation result, $\left(\overline{B_{r}^{\text {total }}}\right)$ requires $6.9 \mathrm{G}$, while $\overline{B_{r}^{\text {dipole }}}$ is $2.64 \mathrm{G}$ obtained from the geomagnetic model, therefore, $\overline{B_{r}^{\text {uniform }}}$ should be $6.4 \mathrm{G}$.

[124] In order to compare the magnetic torque on mantle $\left(\boldsymbol{\Gamma}_{P 1}\right)$ in paper 1 and $\boldsymbol{\Gamma}_{P 2}$ in paper 2 , and therefore the coupling constants $K_{P 1}^{c m b}$ and $K_{P 2}^{c m b}$, we also calculate them for two fields: (1) dipole and (2) uniform.

\section{C1. Dipole Field: $B_{0}^{r}(r, \theta, \phi)=B_{p} \cos \theta$}

[125] For paper 1

$$
\begin{aligned}
A_{1} & =\int_{S}\left[B_{0}^{r}\right]^{2}\left(r^{2} \boldsymbol{\Omega}_{f}\right) d S=\left(a_{c}\right)^{2}\left(B_{p}\right)^{2} \boldsymbol{\Omega}_{f}\left[\int_{S}[\cos \theta]^{2} d S\right] \\
& =\frac{4}{3} \pi\left(a_{c}\right)^{4}\left(B_{p}\right)^{2} \boldsymbol{\Omega}_{f}
\end{aligned}
$$

and

$$
A_{2}=\int_{S}\left[B_{0}^{r}\right]^{2}\left(\mathbf{r} \cdot \Omega_{f}\right) \mathbf{r} d S=\frac{4}{15} \pi\left(a_{c}\right)^{4}\left(B_{p}\right)^{2} \Omega_{f}
$$

therefore,

$$
\Gamma_{P 1}=\frac{k}{\mu_{0}}\left(A_{1}-A_{2}\right)=\frac{16 \pi}{15} \frac{k}{\mu_{0}}\left(a_{c}\right)^{4}\left(B_{p}\right)^{2} \Omega_{f} .
$$

[126] On the other hand, from the results of paper 2, the magnetic torque at boundary $\left(r=a_{b}\right)$ can be expressed as

$$
\tilde{\Gamma}=\gamma_{b} I_{b} \Delta \tilde{m},
$$

where

$$
\gamma_{b}=\frac{-i \pi a_{b}^{4} \Omega_{0}}{2 \mu_{0}\left(\sqrt{\eta_{f}}+\sqrt{\eta_{s}}\right) \sqrt{2|\omega|}}
$$

and for the weak-field approximation,

$$
I_{b}=-2(i+\omega /|\omega|) \int_{0}^{\pi}\left(B_{0}^{r}\right)^{2}\left(1+\cos ^{2} \theta\right) \sin \theta d \theta .
$$

[127] If we also approximate $B_{0}^{r}=B_{p} \cos \theta$ and having $\omega /|\omega|=1$,

$$
\begin{aligned}
I_{b} & =-2(i+\omega /|\omega|) \int_{0}^{\pi}\left(B_{0}^{r}\right)^{2}\left(1+\cos ^{2} \theta\right) \sin \theta d \theta \\
& =\frac{-32(1+i)}{15}\left(B_{p}\right)^{2}
\end{aligned}
$$

then at $\mathrm{CMB}\left(r=a_{c}\right)$

$$
\begin{aligned}
\Gamma_{P 2} & =\gamma_{b} I_{b} \Delta \tilde{m} \\
& =\frac{-32(1+i)}{15}\left(B_{p}\right)^{2} \frac{-i \pi a_{c}^{4} \Omega_{0}}{2 \mu_{0}\left(\sqrt{2|\omega| \eta_{f}}+\sqrt{2|\omega| \eta_{s}}\right)} \Delta \tilde{m} .
\end{aligned}
$$

[128] Using equation (27) of our paper, $k_{2}=\frac{(1-i)}{\sqrt{2 \eta_{m}|\omega|}+\sqrt{2 \eta_{f}|\omega|}}$, for the coefficient $-k$ here, and the definition of $\Delta \tilde{m}$ in paper 2 and $\boldsymbol{\Omega}_{f}$ in paper 1 , one can see that $\boldsymbol{\Gamma}_{P 2}=\boldsymbol{\Gamma}_{P 1}$ for dipole field.

\section{C2. Uniform Field: $B_{0}^{r}(r, \theta, \phi)=B_{u}$}

[129] For paper 1

$A_{1}=\int_{S}\left[B_{0}^{r}\right]^{2}\left(r^{2} \Omega_{f}\right) d S=\left(a_{c}\right)^{2}\left(B_{u}\right)^{2} \Omega_{f}\left[\int_{S} d S\right]=4 \pi\left(a_{c}\right)^{4}\left(B_{u}\right)^{2} \Omega_{f}$

and

$$
\begin{aligned}
A_{2} & =\int_{S}\left[B_{0}^{r}\right]^{2}\left(\mathbf{r} \cdot \boldsymbol{\Omega}_{f}\right) \mathbf{r} d S=\left(B_{u}\right)^{2} \int_{S}\left(\mathbf{r} \cdot \boldsymbol{\Omega}_{f}\right) \mathbf{r} d S \\
& =\frac{4}{3} \pi\left(a_{c}\right)^{4}\left(B_{u}\right)^{2} \boldsymbol{\Omega}_{f},
\end{aligned}
$$

where it is easy to write out the last integral by its three components (or using Gauss theorem to transform this close surface integral to a volume integral). Therefore,

$$
\boldsymbol{\Gamma}_{P 1}=\frac{k}{\mu_{0}}\left(A_{1}-A_{2}\right)=\frac{8 \pi}{3} \frac{k}{\mu_{0}}\left(a_{c}\right)^{4}\left(B_{u}\right)^{2} \boldsymbol{\Omega}_{f} .
$$


[130] On the other hand, for paper 2, if also approximate $B_{0}^{r}=B_{u}$,

$$
\begin{aligned}
I_{b} & =-2(i+\omega /|\omega|) \int_{0}^{\pi}\left(B_{0}^{r}\right)^{2}\left(1+\cos ^{2} \theta\right) \sin \theta d \theta \\
& =\frac{-16(1+i)}{3}\left(B_{u}\right)^{2}
\end{aligned}
$$

then,

$$
\Gamma_{P 2}=\gamma_{b} I_{b} \Delta \tilde{m}=\frac{-16(1+i)}{3} \frac{-i \pi a_{b}^{4} \Omega_{0}\left(B_{u}\right)^{2}}{2 \mu_{0}\left(\sqrt{2|\omega| \eta_{f}}+\sqrt{2|\omega| \eta_{s}}\right)} \Delta \tilde{m}
$$

Again, $\boldsymbol{\Gamma}_{P 2}=\boldsymbol{\Gamma}_{P 1}$ for uniform field.

\section{C3. Summary and Discussion}

[131] According to the statement in paper 2, if $\bar{B}<15 \mathrm{G}$, the weak field approximation is valid. paper 2 adopts $6.9 \mathrm{G}$ for $\overline{B^{\text {total }}}$. Therefore, it is reasonable to calculate $I_{b}$ by using equation (47) of paper 2 which is only valid for weak field approximation.

[132] From the above analysis, we can conclude that, when using the same magnetic field and the weak field approximation, paper 1 and paper 2 should get the same magnetic torque on the mantle, and therefore the same magnetic coupling constant $K^{c m b}$ at CMB.

\section{Appendix D: On the Formula for Lorentz Force}

[133] We will derive the GSSH expression for the Lorentz force $\mathbf{L}$ (equation (34)) for the two approximations of $B_{0}^{r}(r, \theta, \phi)$ as is done in paper 2 , i.e., dipole field and uniform field.

[134] First, we will discuss the contribution, $\mathbf{L}_{p}$ by the dipole field $B_{r}^{\text {dipole }}(r, \theta, \phi)=B_{p} \cos \theta$.

$$
\begin{gathered}
\left(B_{0}^{r}\right)^{2}=\left(B_{p}\right)^{2}(\cos \theta)^{2}=\left(B_{p}\right)^{2}\left(\frac{2}{3} D_{00}^{2}+\frac{1}{3}\right) \\
\mathbf{L}_{p}=\frac{k}{\mu_{0}}\left(B_{0}^{r}\right)^{2} \partial_{r} \mathbf{V}_{f-s} \rightarrow \frac{i \omega k}{\mu_{0}}\left(B_{0}^{r}\right)^{2} \partial_{r} \mathbf{s}_{f-s} \\
=\frac{i \omega k}{\mu_{0}}\left(B_{p}\right)^{2}\left(\frac{2}{3} D_{00}^{2}+\frac{1}{3}\right) \sum_{\alpha=-, 0,+} \sum_{l, m}\left(\partial_{r} S_{l}^{m \alpha}\right)_{f-s} D_{m \alpha}^{l} \hat{e_{\alpha}},
\end{gathered}
$$

where the notation system is identical to the one used by Smith [1974], and

$$
\begin{aligned}
D_{00}^{2} & \sum_{l, m}\left(\partial_{r} S_{l}^{m \alpha}\right)_{f-s} D_{m \alpha}^{l} \\
& =\sum_{l, m}\left\{\sum_{l^{\prime}=|l-2|}^{l+2}\left[\begin{array}{ccc}
l & 2 & l^{\prime} \\
\alpha & 0 & \alpha \\
m & 0 & m
\end{array}\right]\left(\partial_{r} S_{l^{\prime}}^{m \alpha}\right)_{f-s}\right\} D_{m \alpha}^{l}
\end{aligned}
$$

(see Huang [2001, section 4.2] for the detailed derivation of above formula).
[135] To be incorporated into the motion equation and expanded into scalar equations as in the work by Smith [1974], $\mathbf{L}_{p}$ is represented by its three scalar components, radial part $\left[\mathbf{L}_{p}\right]^{U}$, transverse spheroidal part $\left[\mathbf{L}_{p}\right]^{V}$ and toroidal part $\left[\mathbf{L}_{p}\right]^{W}$. According to section 2.3 , only the toroidal term is needed in the calculation, and noting equation (37), we can write out

$$
\begin{aligned}
{\left[\mathbf{L}_{p}\right]_{l m}^{W}=} & 2 \sqrt{2} \frac{i \omega k}{\mu_{0}}\left(B_{p}\right)^{2}\left\{\frac{1}{3}\left(\partial_{r} W_{l}^{m}\right)_{f-s}\right. \\
& \left.+\frac{2}{3} \sum_{l^{\prime}=|l-2|}^{l+2}\left[\begin{array}{ccc}
l & 2 & l^{\prime} \\
+ & 0 & + \\
m & 0 & m
\end{array}\right]\left[\begin{array}{c}
\left(\partial_{r} W_{l^{\prime}}^{m}\right)_{f-s} \\
\left(\partial_{r} V_{l^{\prime}}^{m}\right)_{f-s}
\end{array}\right]\right\} \begin{array}{l}
l-l^{\prime} \mid \text { even } \\
\left|l-l^{\prime}\right| \text { odd }
\end{array}
\end{aligned}
$$

[136] For the uniform field approximation, $B_{0}^{r}(r, \theta, \phi)=B_{u}$, one can more easily write out the toroidal part of its contribution to the Lorentz force:

$$
\left[\mathbf{L}_{u}\right]_{l m}^{W}=2 \sqrt{2} \frac{i \omega k}{\mu_{0}}\left(B_{u}\right)^{2}\left(\partial_{r} W_{l}^{m}\right)_{f-s} .
$$

[137] For simplicity, we use a uniform field approximation here with the RMS value of the total field given in paper 2, i.e., $B_{u}=6.9 G$.

\section{Appendix E: Calculation of $\boldsymbol{G}_{\boldsymbol{l n}}^{\boldsymbol{m k}}$}

[138] $G_{l n}^{m k}$ is used in the calculation of the EMC torque, but its analytic form is unavailable in the literature and not easy to get. However, we can derive it if $l=n$ and $m=k$.

$$
T=G_{l l}^{m m}=\int_{-1}^{1}\left(1-x^{2}\right) P_{l}^{m} P_{l}^{m} d x=D-A,
$$

where

$$
\begin{gathered}
D \equiv \int_{-1}^{1}\left(P_{l}^{m}\right)^{2} d x=\frac{2}{2 l+1} \frac{(l+m) !}{(l-m) !} \\
A \equiv \int_{-1}^{1} x^{2} P_{l}^{m} P_{l}^{m} d x .
\end{gathered}
$$

[139] From the recurrence of $P_{l}^{m}$,

$$
(2 l+1) x P_{l}^{m}=(l+m) P_{l-1}^{m}+(l-m+1) P_{l+1}^{m},
$$

we have

$$
\begin{aligned}
x^{2} P_{l}^{m}= & G_{l}^{m}\left(x P_{l-1}^{m}\right)+H_{l}^{m}\left(x P_{l+1}^{m}\right)=G_{l}^{m}\left[G_{l-1}^{m} P_{l-2}^{m}+H_{l-1}^{m} P_{l}^{m}\right] \\
& +H_{l}^{m}\left[G_{l+1}^{m} P_{l}^{m}+H_{l+1}^{m} P_{l+2}^{m}\right]
\end{aligned}
$$

where

$$
G_{l}^{m}=\frac{l+m}{2 l+1}, \quad H_{l}^{m}=\frac{l-m+1}{2 l+1} .
$$


[140] Using the orthogonality property of $P_{l}^{m}$, we get

$$
\int_{-1}^{1} x^{2} P_{l}^{m} P_{l}^{m} d x=0+\left[G_{l}^{m} H_{l-1}^{m}+H_{l}^{m} G_{l+1}^{m}\right] D+0 ;
$$

therefore,

$$
\begin{aligned}
T & =\left\{1-\left[G_{l}^{m} H_{l-1}^{m}+H_{l}^{m} G_{l+1}^{m}\right]\right\} D \\
& =\left\{1-\frac{(l+m)(l-m)}{(2 l+1)(2 l-1)}-\frac{(l+m+1)(l-m+1)}{(2 l+1)(2 l+3)}\right\} D .
\end{aligned}
$$

[141] Acknowledgments. This work is supported by projects 10773025,11073044 , and 10633030 of NSFC, while the computational work was completed during a 3 month visit of HCL to the Royal Observatory of Belgium, supported by the Services Federaux des Affaires Scientifiques, Techniques et Culturelles of Belgium. The initialing discussion with O. de Viron is appreciated. Two anonymous reviewers and the Associate Editor are also appreciated for their friendly encouragement and constructive suggestions.

\section{References}

Ballani, L., H. Greiner-Mai, and D. Stromeyer (2002), Determining the magnetic field in the core-mantle boundary zone by non-harmonic downward continuation, Geophys. J. Int., 149, 374-389.

Buffett, B. A. (1992), Constraints on magnetic energy and mantle conductivity from the forced nutations of the Earth, J. Geophys. Res., 97(B13), $19,581-19,597$.

Buffett, B. A. (1993), Influence of a toroidal magnetic field on the nutation of Earth, J. Geophys. Res., 98(B2), 2105-2117.

Buffett, B. A. (2007), Core-mantle interactions, in Treatise on Geophysics, vol. 8, Core Dynamics, edited by G. Schubert, pp. 345-358, Elsevier, Amsterdam.

Buffett, B. A., and U. R. Christensen (2007), Magnetic and viscous coupling at the core-mantle boundary: Inferences from observations of the Earth's nutations, Geophys. J. Int., 171, 145-152.

Buffett, B. A., P. M. Mathews, T. A. Herring, and I. I. Shapiro (1993), Forced nutation of the Earth: Contributions from the effects of ellipticity and rotation on the elastic deformations, J. Geophys. Res., 98(B12), 21,659-21,676.

Buffett, B. A., E. J. Garnero, and R. Jeanloz (2000), Sediments at the top of the Earth's core, Science, 290, 1338-1342.

Buffett, B. A., P. M. Mathews, and T. A. Herring (2002), Modeling of nutation and precession: Effects of electromagnetic coupling, J. Geophys. Res., 107(B4), 2070, doi:10.1029/2000JB000056.

Buffett, B. A., J. Mound, and A. Jackson (2009), Inversion of torsional oscillations for the structure and dynamics of Earth's core, Geophys. J. Int., 177, 878-890.

Capitaine, N., J. Chapront, S. Lambert, and P. T. Wallance (2003), Expressions for the celestial intermediate pole and celestial ephemeris origin consistent with the IAU 2000 A precession-nutation model, Astron. Astrophys., 400, 1145-1154, doi:10.1051/0004-6361:20030077.

Christensen, U. R., and J. Wicht (2007), Numerical dynamo simulations, in Treatise on Geophysics, vol. 8, Core Dynamics, edited by G. Schubert, pp. 245-282, Elsevier, Amsterdam.

Dahlen, F. A., and J. Tromp (1998), Theoretical Global Seismology, Princeton Univ. Press, Princeton, N. J.

Dehant, V. (1990), On the nutations of a more realistic Earth's model, Geophys. J. Int., 100, 477-483.

Dehant, V., and P. Defraigne (1997), New transfer functions for nutations of a nonrigid Earth, J. Geophys. Res., 102(B12), 27,659-27,687.

Deleplace, B., and P. Cardin (2006), Visco-magnetic torque at the core mantle boundary, Geophys. J. Int., 167, 557-566.

Dumberry, M., and J. E. Mound (2008), Constraints on core-mantle electromagnetic coupling from torsional oscillation normal modes, J. Geophys. Res., 113, B03102, doi:10.1029/2007JB005135.

Folgueira, M., and V. Dehant (2008), Estimation of the topographic torque at the core-mantle boundary on nutation, in Proceedings of "Journées Systèmes de Référence Spatio-Temporels 2007, ” edited by N. Capitaine, p. 115, Obs. de Paris, Paris.

Getino, J. (1995), Forced nutations of a rigid mantle-liquid core Earth model in canonical formulation, Geophys. J. Int., 122, 803-814.

Getino, J., and J. M. Ferrándiz (2001), Forced nutations of a two-layer Earth model, Mon. Not. R. Astron. Soc., 322, 785-799.
Glatzmaier, G. A. (2002), Geodynamo simulations-How realistic are they?, Annu. Rev. Earth Planet. Sci., 30, 237-257, doi:10.1146/annurev. Earth.30.091201.140817.

Greenspan, H. P. (1968), The Theory of Rotating Fluids, Cambridge Univ. Press, New York.

Gubbins, D., and P. H. Roberts (1987), Magnetohydrodynamics of the Earth's core, in Geomagnetism, vol. 2, edited by J. A. Jacobs, pp. 1-183, Academic, San Diego, Calif.

Herring, T. A., B. A. Buffett, P. M. Mathews, and I. I. Shapiro (1991), Forced nutations of an Earth: Influence of inner core dynamics: 3. Very long interferometry data analysis, J. Geophys. Res., 96(B5), 8259-8273.

Herring, T. A., P. M. Mathews, and B. A. Buffett (2002), Modeling of nutation-precession: Very long baseline interferometry results, J. Geophys. Res., 107(B4), 2069, doi:10.1029/2001JB000165.

Holme, R. (1998), Electromagnetic core-mantle coupling-I. Explaining decadal changes in the length of day, Geophys. J. Int., 132, 167-180.

Holme, R. (2000), Electromagnetic core-mantle coupling-III. Laterally varying mantle conductance, Phys. Earth Planet. Inter, 117, 329-344.

Huang, C. L. (2001), The scalar boundary conditions for the motion of the elastic Earth to second order in ellipticity, Earth Moon Planets, 84(3), $125-141$.

Huang, C. L., W. J. Jin, and X. H. Liao (2001), A new nutation model of a non-rigid Earth with ocean and atmosphere, Geophys. J. Int., 146 $126-133$

Huang, C. L., V. Dehant, and X. H. Liao (2004), The explicit scalar equations of infinitesimal elastic-gravitational motion in the rotating, slightly elliptical fluid outer core of the Earth, Geophys. J. Int., 157, 831-837.

Huang, C. L., V. Dehant, X. H. Liao, O. de Viron, and T. Van Hoolst (2005), The coupling equations between the nutation and the geomagnetic field in GSH expansion, in Fundamental Astronomy: New Concepts and Models for High Accuracy Observations? Proceedings of Journées Systèmes de Référence Spatio-Temporels 2004, edited by N. Capitaine, pp. 88-93, Obs. de Paris, Paris.

International Association of Geomagnetism and Aeronomy Division V, Working Group 8 (2000), International Geomagnetic Reference Field 2000, Geophys. J. Int., 141, 259-262.

Jackson, A., and P. Livermore (2009), On Ohmic heating in the Earth's core I: Nutation constraints, Geophys. J. Int., 177, 367-382.

Kuang, W., and J. Bloxham (1999), Numerical modeling of magnetohydrodynamic convection in a rapidly rotating spherical shell: Weak and strong field dynamo action, J. Comput. Phys., 153, 51-81.

Mathews, P. M., and J. Y. Guo (2005), Viscoelectromagnetic coupling in precession-nutation theory, J. Geophys. Res., 110, B02402, doi:10.1029/ 2003JB002915.

Mathews, P. M., T. A. Herring, B. A. Buffett, and I. I. Shapiro (1991), Forced nutation of the Earth: Influence of the inner core dynamics. 1: Theory, J. Geophys. Res., 96(B5), 8219-8242.

Mathews, P. M., T. A. Herring, and B. A. Buffett (2002), Modeling of nutation and precession: New nutation series for nonrigid Earth and insights into the Earth's interior, J. Geophys. Res., 107(B4), 2068, doi:10.1029/ 2001JB000390.

Melchior, P. J. (1986), The Physics of the Earth's Core: An Introduction, Pergamon, Oxford, U. K.

Moffatt, H. K. (1978), Magnetic Field Generation in Electrically Conducting Fluids, Cambridge Univ. Press, New York.

Mound, J. E., and B. A. Buffett (2005), Mechanicms of core-mantle angular momentum exchange and the observed spectral properties of torsional oscillations, J. Geophys. Res., 110, B08103, doi:10.1029/2004JB003555.

Mound, J., and B. Buffett (2007), Viscosity of the Earth's fluid core and torsional oscillations, J. Geophys. Res., 112, B05402, doi:10.1029/ 2006JB004426.

Palmer, A., and D. Smylie (2005), VLBI observations of free core nutations and viscosity at the top of the core, Phys. Earth Planet. Inter., 148, 285-301.

Rochester, M. G. (1962), Geomagnetic core-mantle coupling, J. Geophys Res., 67(12), 4833-4836.

Rochester, M. G. (1976), The secular decrease of obliquity due to dissipative core-mantle coupling, Geophys. J. R. Astron. Soc., 46, 109-126.

Rochester, M. G., and D. J. Crossley (2009), Earth's long-period wobbles: A Lagrangean description of the Liouville equations, Geophys. J. Int., $176,40-62$

Rochester, M. G., and D. E. Smylie (1965), Geomagnetic core-mantle coupling and the Chandler wobble, Geophys. J. R. Astron. Soc., 10, 289-315.

Rogister, Y. (2001), On the diurnal and nearly diurnal free modes of the Earth, Geophys. J. Int., 144, 459-470.

Rogister, Y. (2003), Splitting of seismic-free oscillations and of the Slichter triplet using the normal mode theory of a rotating, ellipsoidal Earth, Phys. Earth Planet. Inter., 140, 169-182. 
Rogister, Y., and B. Valette (2009), Influence of liquid core dynamics on rotational modes, Geophys. J. Int., 176, 368-388, doi:10.1111/j.1365246X.2008.03996.x.

Sasao, T., S. Okubo, and M. Saito (1980), A simple theory on the dynamical effects of a stratified fluid core upon nutational motion of the Earth, in Proceedings of IAU Symposium 78, edited by E. P. Fedrov, M. L. Smith, and P. L. Bender, pp. 165-183, D. Reidel, Hingham, Mass.

Schastok, J. (1997), A new nutation series for a more realistic model Earth, Geophys. J. Int., 130, 137-150.

Smith, M. L. (1974), The scalar equations of infinitesimal elasticgravitational motion for a rotating, slightly elliptical Earth, Geophys. J. R. Astron. Soc., 37, 491-526.

Stix, M., and P. H. Roberts (1984), Time-dependent electromagnetic coremantle coupling, Phys. Earth Planet. Inter., 36, 49-60.

Wahr, J. M. (1981), The forced nutations of an elliptical, rotating, elastic and oceanless Earth, Geophys. J. R. Astron. Soc., 64, 705-727.
Wahr, J., and J. de Vries (1989), The possibility of lateral structure inside the core and its implications for nutation and Earth tides observations, Geophys. J. Int., 99, 511-519.

Wicht, J., and D. Jault (1999), Constraining electromagnetic core-mantle coupling, Phys. Earth Planet. Inter., 111, 161-177.

Wu, X. P., and J. Wahr (1997), Effects of non-hydrostatic core-mantle boundary topography and core dynamics on Earth rotation, Geophys. J. Int., $128,18-42$.

V. Dehant and T. Van Hoolst, Royal Observatory of Belgium, Ave. Circulaire 3,B-1180 Brussels, Belgium. (v.dehant@oma.be; tim. vanhoolst@oma.be)

C.-L. Huang and X.-H. Liao, Shanghai Astronomical Observatory, Chinese Academy of Sciences, 80 Nandan Rd., Shanghai 200030, China. (clhuang@shao.ac.cn; xhliao@shao.ac.cn)

M. G. Rochester, Department of Earth Sciences, Memorial University of Newfoundland, St. John's, NL A1B 3X5, Canada. (mrochest@mun.ca) 